\title{
Investigation of Fluid-Structure-Coupling and Turbulence Model Effects on the DLR Results of the Fifth AIAA CFD Drag Prediction Workshop
}

\author{
Stefan Keye* $\quad$ Vamshi Togiti ${ }^{\dagger} \quad$ Bernhard Eisfeld ${ }^{\ddagger}$ \\ Olaf P. Brodersen ${ }^{\S}$ \\ DLR, German Aerospace Center, \\ Institute of Aerodynamics and Flow Technology, 38108 Braunschweig, Germany \\ Melissa B. Rivers \\ NASA Langley Research Center, Hampton, VA, 23681, USA
}

\section{Nomenclature}

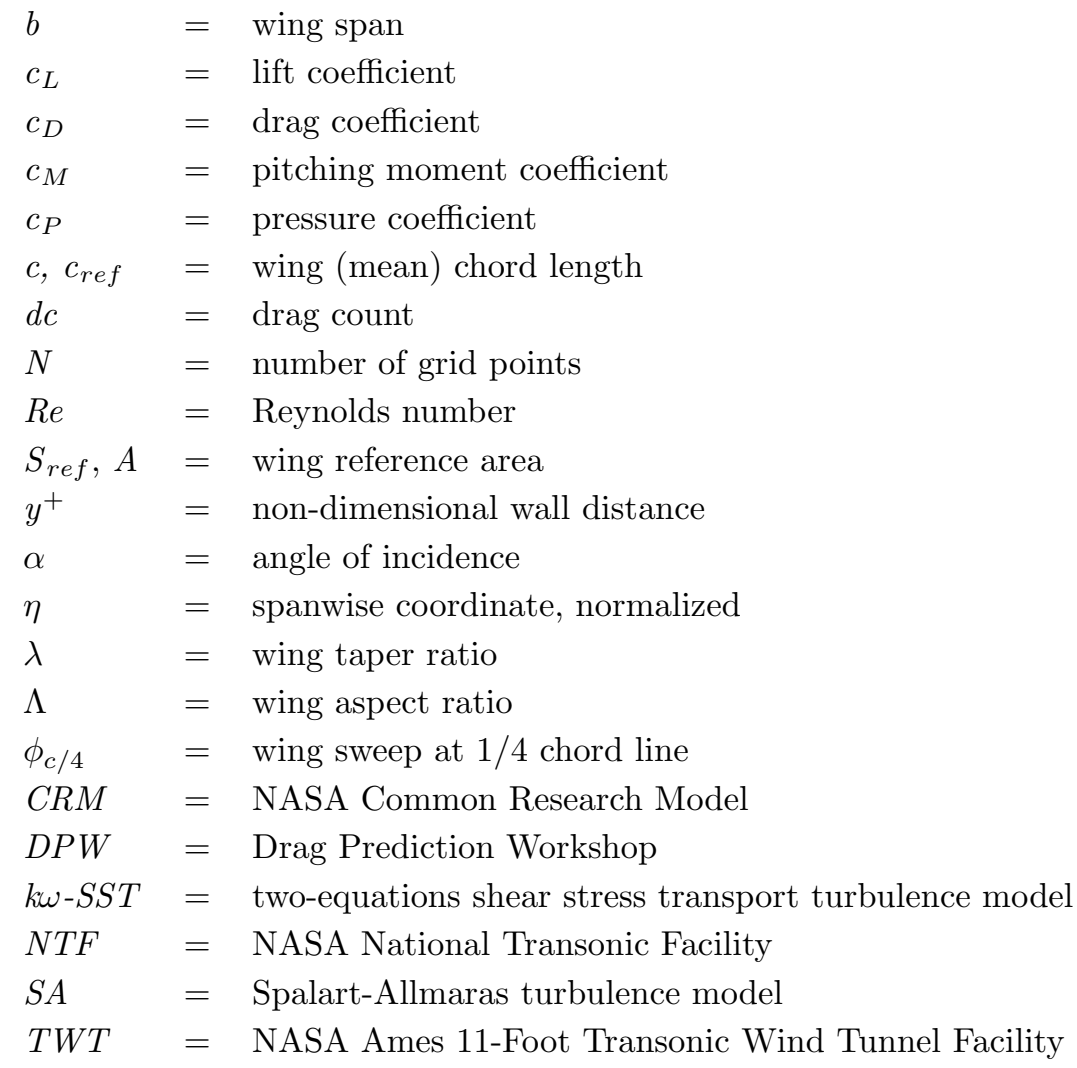

${ }^{*}$ Research Scientist, Dept. Transport Aircraft.

${ }^{\dagger}$ Research Scientist, Dept. CASE.

${ }^{\ddagger}$ Research Scientist, Dept. CASE.

$\S$ Research Scientist, Dept. Transport Aircraft, Member AIAA.

IResearch Engineer, Configuration Aerodynamics Branch, Mail Stop 267, Senior Member AIAA. 


\section{Introduction}

7 HE accurate calculation of aerodynamic forces and moments is of significant importance during the design 1 phase of an aircraft. Reynolds-averaged Navier-Stokes (RANS) based Computational Fluid Dynamics (CFD) has been strongly developed over the last two decades regarding robustness, efficiency, and capabilities for aerodynamically complex configurations. ${ }^{1,2}$ Incremental aerodynamic coefficients of different designs can be calculated with an acceptable reliability at the cruise design point of transonic aircraft for non-separated flows. But regarding absolute values as well as increments at off-design significant challenges still exist to compute aerodynamic data and the underlying flow physics with the accuracy required.

In addition to drag, pitching moments are difficult to predict because small deviations of the pressure distributions, e.g. due to neglecting wing bending and twisting caused by the aerodynamic loads can result in large discrepancies compared to experimental data. Flow separations that start to develop at off-design conditions, e.g. in corner-flows, at trailing edges, or shock induced, can have a strong impact on the predictions of aerodynamic coefficients too.

Based on these challenges faced by the CFD community a working group of the AIAA Applied Aerodynamics Technical Committee initiated in 2001 the CFD Drag Prediction Workshop (DPW) series resulting in five international workshops. The results of the participants and the committee are summarized in more than 120 papers. ${ }^{3-7}$ The latest, fifth workshop took place in June 2012 in conjunction with the 30th AIAA Applied Aerodynamics Conference. ${ }^{8}$

All workshops were focused on the following key objectives: ${ }^{6}$

- assess state-of-the-art CFD methods as practical aerodynamic tools for the accurate prediction of forces and moments on industry-relevant aircraft configurations, with a focus on absolute as well as incremental values,

- setup an international forum of experts from industry, research and academia for the verification and validation of RANS based CFD methods by applying different meshing methods and turbulence models,

- define areas for additional research needed,

- build, use, and maintain a public-domain transonic flow database for transport aircraft geometries including CAD data, grids, and numerical and experimental results,

- document workshop findings and to disseminate through presentations and publications.

NASA and the DLR Institute of Aerodynamics and Flow Technology are supporting these objectives as committee members and participants. ${ }^{9-13}$

The first three workshops used DLR transonic wind tunnel model configurations and experimental data achieved together with ONERA. ${ }^{3-5,14,15}$ For the fourth and fifth workshops a new configuration the socalled Common Research Model (CRM) was defined by NASA and Boeing ${ }^{16}$ for transonic flow conditions, see figure 1. In 2009/2010 experimental wind tunnel campaigns were performed with the CRM by NASA in the National Transonic Facility (NTF) at Langley and in the Ames 11-ft tunnel. The data have been published recently in several papers. ${ }^{17-19}$

A major aspect came into focus when the DPW-4 and DPW-5 computational results of the participants have been compared to the experimental data. Besides moderate discrepancies in drag at the cruise design point significant offsets of the pitching moments have been observed. These have been traced back to the model support system, which extends vertically from the aft fuselage, and to a deviation of wing twist between the computational and the wind tunnel model geometries. ${ }^{20,21}$

DLR results in DPW-4 and DPW-5 also showed differences between experimental data and numerical calculations of the wing pressure distributions, especially for the most outboard sections as presented in Figs. 2 and 3 nearly independent of the different grid refinement levels (L2: coarse, L6: ultra fine). Therefore, it is the first objective of these investigations to evaluate the influence of static aeroelastic wing deformations onto pressure distributions and overall aerodynamic coefficients. NASA and DLR decided to perform, in addition to their other investigations, fluid-structure-coupled simulations based on the NASA finite-element structural model of the CRM wind tunnel model and the DLR TAU CFD solver.

A second aspect of DLR results in DPW-5 has been the prediction of a small flow separation $(<1 \%$ local chord length) at cruise conditions in the wing-fuselage junction near the wing trailing edge. The size of 


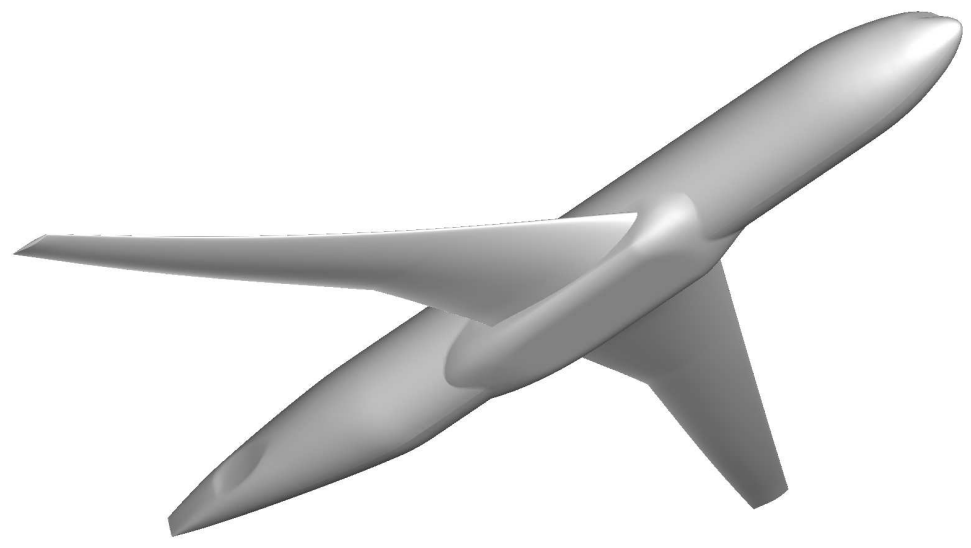

Figure 1. NASA Common Research Model (CRM) without horizontal tail.

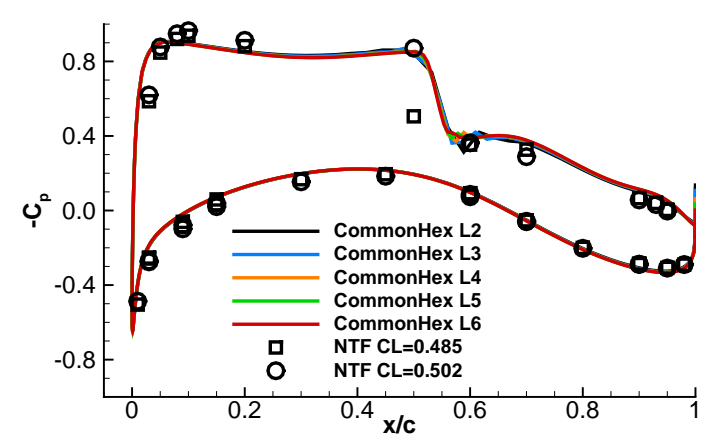

(a) $\eta=0.501$

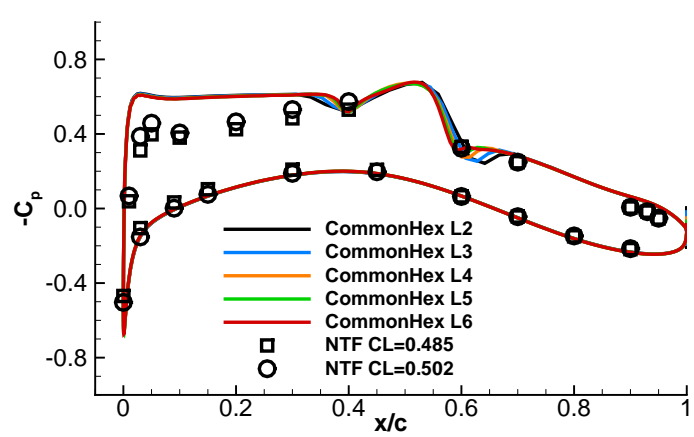

(b) $\eta=0.95$

Figure 2. Pressure distributions, TAU results for common grids (L2: coarse, L6: ultra fine), SA model, NASA NTF test data, Case 1.

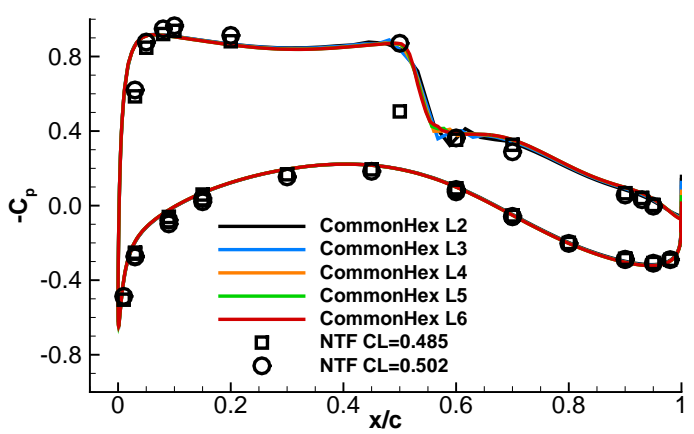

(a) $\eta=0.501$

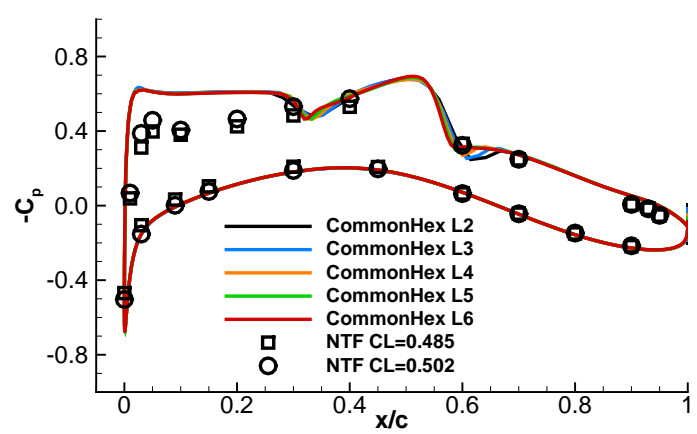

(b) $\eta=0.95$

Figure 3. Pressure distributions, TAU results for common grids (L2: coarse, L6: ultra fine), Menter k $\omega$-SST model, NASA NTF test data, Case 1. 
that separation and its development for increasing incidence angles was found to depend on grid resolution, topology, turbulence modelling, and numerical dissipation as demonstrated in Figs. 4 and 5 . The effect is more pronounced for the Spalart-Allmaras turbulence model than for the Menter k $\omega$-SST model. For very fine grid levels of both the hexahedral common grids and the DLR unstructured hex-dominant grids with overlapping hexahedral element corner block, separation starts close to the location of critical pressure in the corner. Therefore the second objective of these investigations is to analyse how the prediction of the developing corner flow changes, when a higher fidelity turbulence models that take anisotropy into account are applied. Therefore the second objective of these investigations is to analyse how the prediction of the corner flow changes for increasing incidence angles when the SA and the kw-SST turbulence models with and without QCR extension as well as a Reynolds stress model are used.

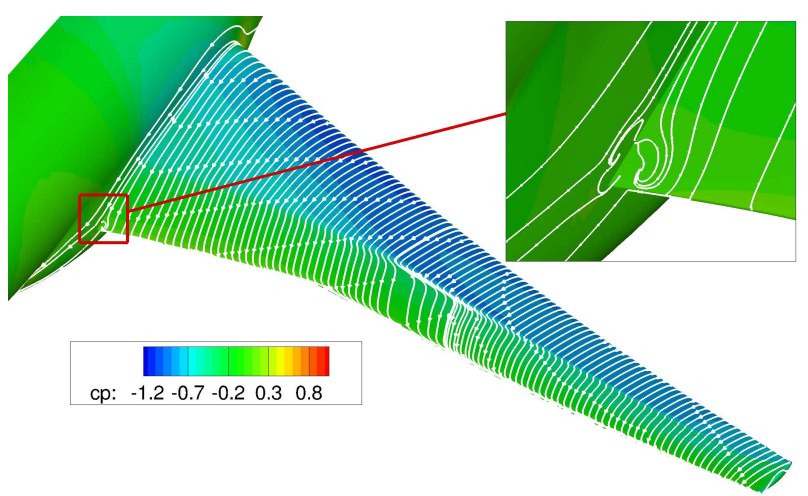

(a) Overview

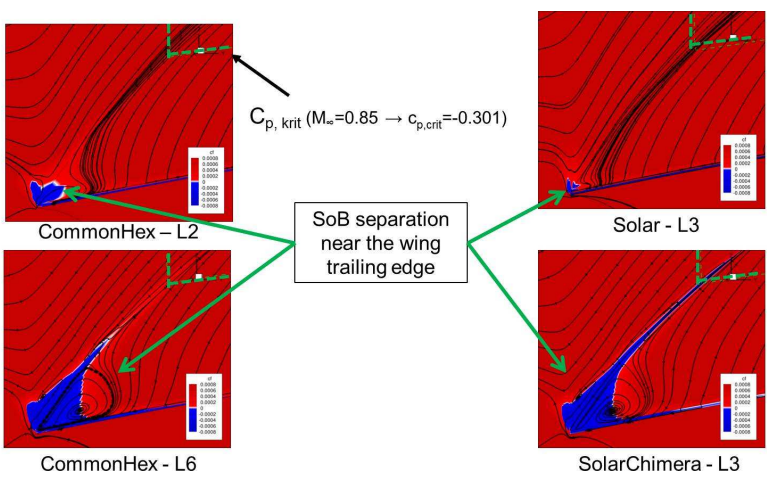

(b) Details, influence of grid resolution and topology, blue: negative $c_{f}$, red: positive $c_{f}$

Figure 4. Side-of-body flow separation at cruise design, $M_{\infty}=0.85, \alpha=3.75^{\circ}, R e=5 \cdot 10^{6}$.
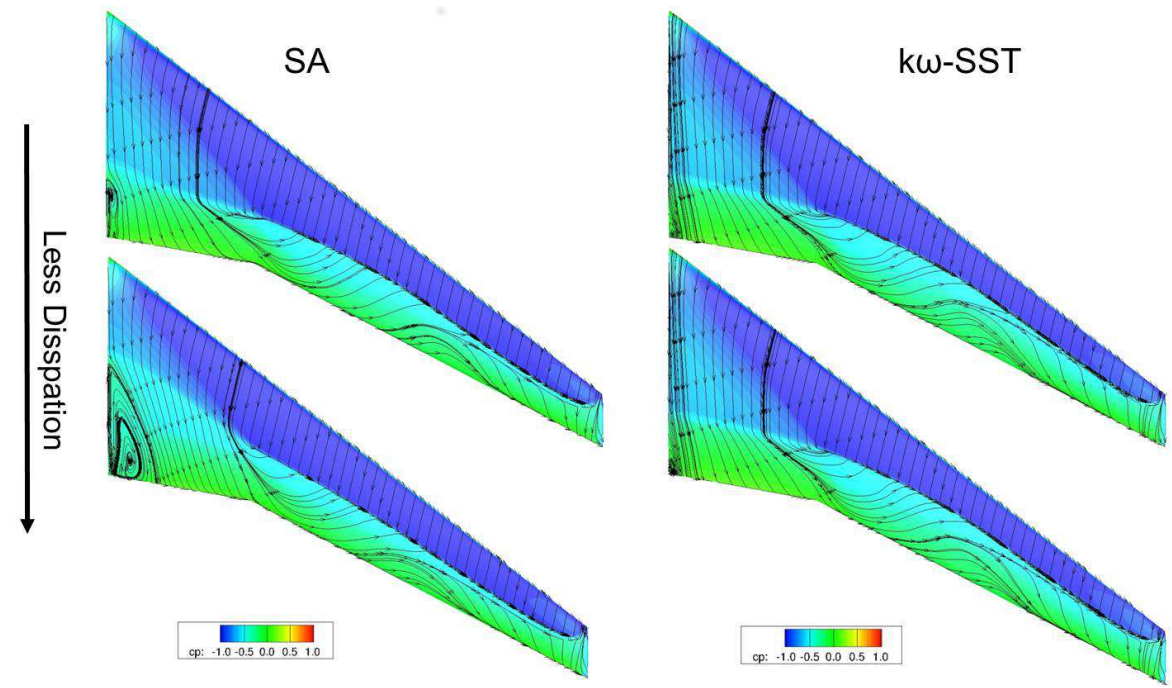

Figure 5. Influence of dissipation and turbulence models on CRM side-of-body flow separation, $M_{\infty}=0.85$, $\alpha=3.75^{\circ}, R e=5 \cdot 10^{6}$

\section{NASA Common Research Model and DPW-5 Test Cases}

For DPW-5 the NASA Common Research Model (CRM) civil transport aircraft configuration for cruise flight conditions $\left(M_{\infty}=0.85, C_{L}=0.5\right.$, altitude $\left.H=11300 \mathrm{~m}\right)$ is used as the reference geometry. The CRM optionally has a horizontal stabilizer as well as engines and pylons. In DPW-5 only the wing-body configuration is used as presented in figure 1. The CRM was designed by NASA's Subsonic Fixed Wing 
Technical Working Group and by Vassberg et al. ${ }^{16}$ The wing has a slightly stronger pressure recovery at the last $10-15 \%$ local chord on the upper surface of the outboard wing section. The objective of this feature is to reduce boundary layer strength to control the development of a trailing edge separation and to create a challenge for turbulence models. The main geometrical features of the CRM are listed in table 1. Further details are published by Vassberg. ${ }^{16}$ The geometrical and experimental data of the model can be found on the NASA CRM web site. ${ }^{22}$

Table 1. CRM geometrical data.

\begin{tabular}{lrrr}
\hline \hline$S_{\text {ref }}$ & $=$ & 383.69 & $\mathrm{~m}^{2}$ \\
$b$ & $=$ & $58.763 \mathrm{~m}$ \\
$c_{r e f}$ & $=$ & $4.8978 \mathrm{~m}$ \\
$\Lambda$ & $=$ & 9.0 & \\
$\varphi_{c / 4}$ & $=$ & $35.0^{\circ}$ & \\
\hline \hline
\end{tabular}

\section{A. DPW-5 Cases}

DPW-5 required two mandatory test cases as a minimum. Additional parameter, grid, and turbulence model variations were allowed.

\section{Case 1, Common Grid Study:}

- Flow conditions: $M_{\infty}=0.85, C_{L}=0.5 \pm 0.0001, R e=5 \cdot 10^{6}$

- Grids: sequence of common grids, custom grids

\section{Case 2, Buffet Study:}

- Flow conditions: $M_{\infty}=0.85, R e=5 \cdot 10^{6}$, steady flow simulations

- $\alpha=\left[2.5^{\circ}, 2.75^{\circ}, 3.0^{\circ}, 3.25^{\circ}, 3.5^{\circ}, 3.75^{\circ}, 4.0^{\circ}\right]$

- Grids: medium (L3) common grid, custom grid

\section{Numerical Methods}

\section{A. TAU CFD Solver}

Since the mid 1990s the Reynolds-averaged Navier-Stokes solver TAU is under development at DLR. It can be traced back to the German CFD project MEGAFLOW which integrated developments of DLR, aircraft industry, and universities. ${ }^{23-25}$ Today the software package is under continuous development by the $\mathrm{C}^{2} \mathrm{~A}^{2} \mathrm{~S}^{2} \mathrm{E}$ department (Center for Computer Applications in AeroSpace Science and Engineering) of the institute and it is applied by DLR, European partners in industry and academia.

TAU is an edge-based unstructured solver using the dual grid technique and fully exploits the advantages of hybrid grids. The numerical scheme is based on the Finite-Volume method and provides different spatial discretization schemes like central and upwind. ${ }^{25}$ Here, a central scheme of second order accuracy, using the Jameson-type of artificial dissipation in scalar and matrix mode, has been applied. ${ }^{26,27}$ Time integration has been performed using both, the explicit Runge-Kutta multistage and the Lower-Upper Symmetric GaussSeidel (LU-SGS) schemes. TAU has been developed with a particular focus on industrial aeronautical applications, thus providing techniques like overlapping grids for treating unsteady phenomena and complex geometries. Further details of TAU can be found in the reference. ${ }^{25}$ 


\section{B. Fluid-Structure-Coupled Simulation Procedure}

DLR's fluid-structure-coupled (FSC) steady state simulation procedure, figure 6, incorporates the in-house flow solver TAU and the computational structural mechanics (CSM) code NASTRAN ${ }^{\circledR}$ as main components. Additional modules included are a bi-directional interpolation routine for mapping aerodynamic loads to the structural nodes and transferring structural deflections back to the CFD mesh, and - closing the coupling loop - a volume mesh deformation algorithm.

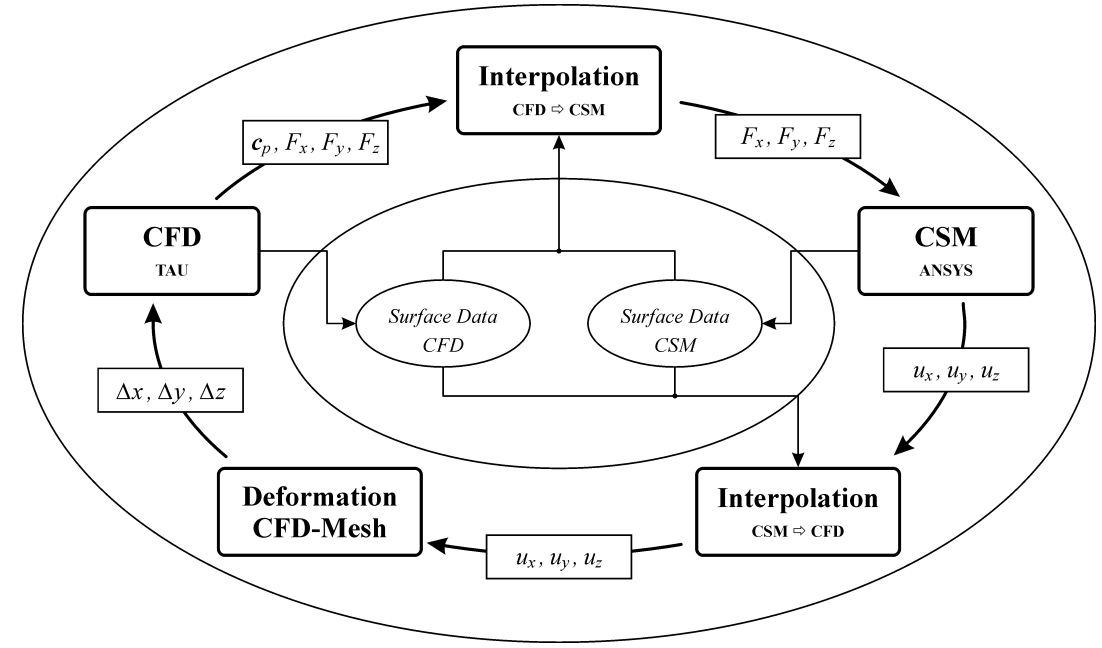

Figure 6. Simulation procedure for fluid-structure-coupled analyses.

The analysis starts from an initial RANS CFD solution, which is computed on the undeformed grid. Then, static pressure and friction coefficients along with the identifiers, coordinates, and connectivity of the grid nodes, which constitute the CFD coupling surface, are transferred to the interpolation module using the Aerodynamic Mesh Interface Format (AMIF) specification.

For each surface element in the CFD grid the interpolation module computes a force vector using pressure coefficient values, cell face area and cell orientation. Then, aerodynamic forces are mapped to the structural nodes located on the coupling surface. The corresponding finite-element surface data is provided from another AMIF file and processed in the same manner. Due to the considerable resolution difference, which usually exists between CFD and structural meshes, or when, as in this case, connectivity data of the finite-element surface nodes is not available, the application of a simple linear interpolation strategy is not applicable and a nearest neighbor search algorithm is used instead. ${ }^{28}$ An assessment of both interpolation methods with respect to the coupling of aerodynamic forces between CFD and structural meshes is provided in. ${ }^{29}$ For a given CFD face centroid $i$ the nearest neighboring CSM grid point $j$ is identified and a force component $F_{j, C S M}$ and associated moment $M_{j, C S M}=F_{i, C F D} \times r_{i j}$ are mapped to node $j$, figure 7. This procedure ensures a conservative interpolation with respect to both force and moment balance on CFD and CSM side. An example showing a computed $c_{p}$-distribution and the equivalent structural force distribution is given in figure 8 (a).
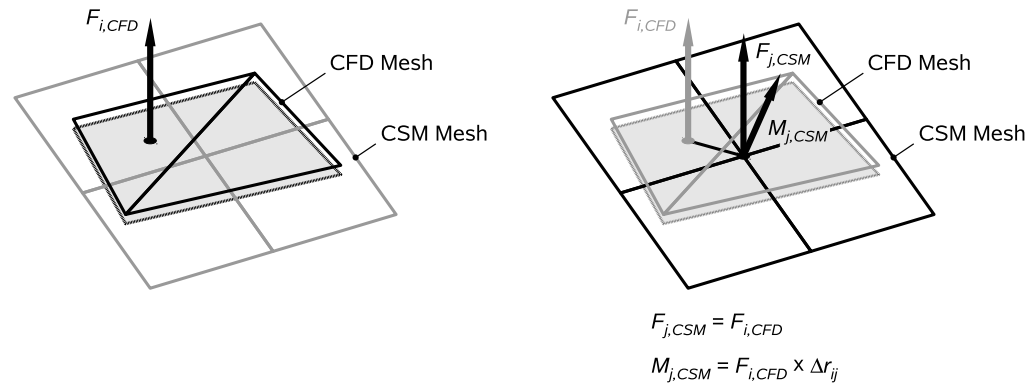

Figure 7. Force mapping between CFD and CSM meshes. 


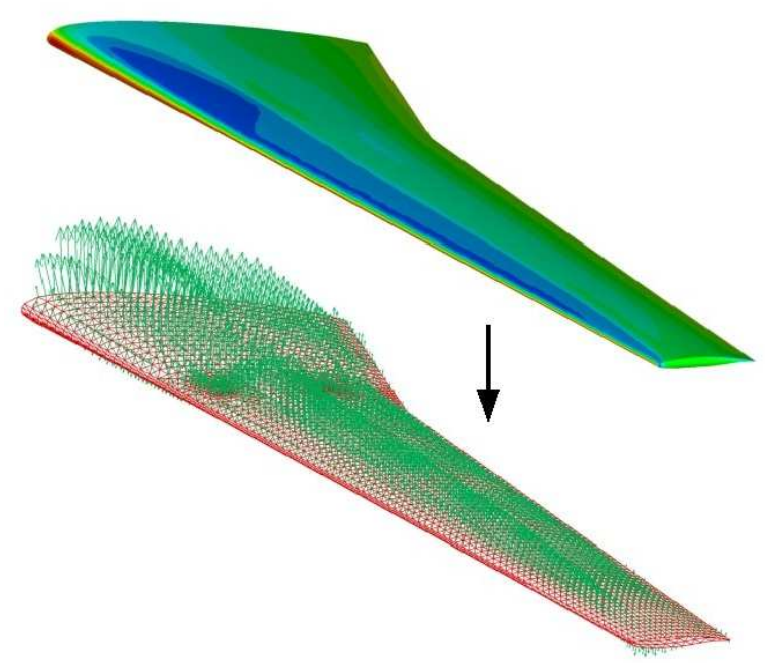

(a) Interpolation of static surface pressure (top) to nodal forces (bottom)

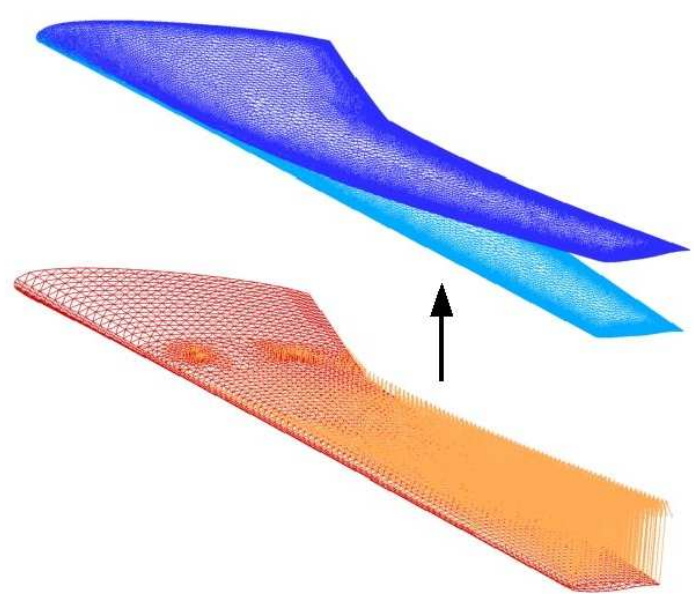

(b) Interpolation of structural deflections (bottom) to CFD surface mesh (top)

Figure 8. Interpolation of aerodynamic loads and structural deflections.

Next, nodal loads from the interpolation routine are re-formatted into NASTRAN ${ }^{\circledR}$ force cards and linked to the bulk data file. A linear, static structural analysis is performed and the resulting nodal deflection components along the coupling surface are mapped back to the CFD surface mesh, figure 8 (b). Because the nearest neighbor search algorithm used before is not appropriate for deformation fields, an interpolation scheme based on radial basis functions (RBF) is used. ${ }^{28}$ The technique is particularly well suited for smooth functions, ${ }^{30,31}$ like the deformations of aerodynamic structures considered in this application.

Before a new flow solution is started, the interpolated surface nodal deflections are extrapolated into the volume mesh. This is achieved by applying the RBF interpolation functions used for the surface mesh deformation to the volume mesh nodes also. Additionally, the resulting deflections are superimposed with a weighting function based on wall distance in order to achieve a gradual decline of nodal deflections from the coupling surface into the flow field and to let them vanish for a specified distance, for example along the farfield boundaries. The method is applicable to both hybrid unstructured and block-structured meshes.

Finally, a new CFD solution is computed on the deformed mesh. A typical convergence history for a fluidstructure-coupled simulation is plotted in figure 9. The individual coupling steps are easily identified by steep increases in density residual and altered lift, drag, and pitching moment coefficient values. Iteration proceeds until user-defined convergence criteria, based on either flow or structural parameters, are accomplished.

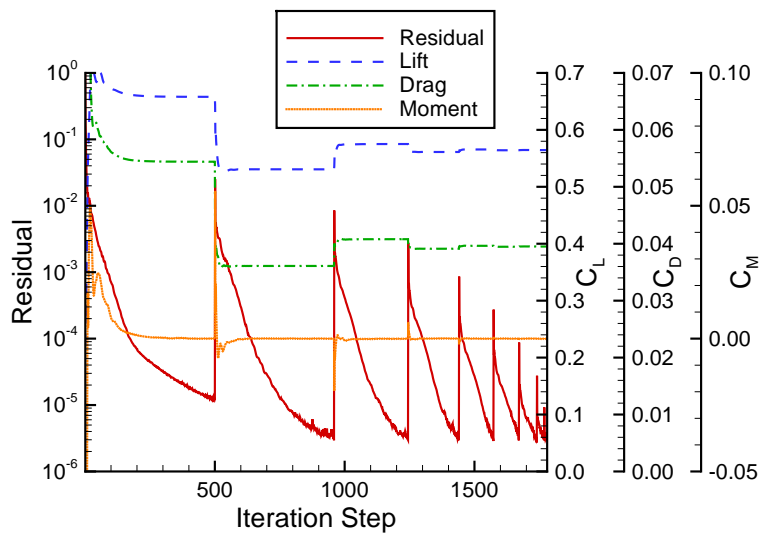

Figure 9. Coupled simulation convergence history. 
DLR's fluid-structure-coupled simulation approach has been validated using a variety of test cases and flow conditions, including both wind tunnel ${ }^{32}$ and flight test data. ${ }^{33}$

\section{Turbulence Modeling}

The DLR TAU code offers various turbulence models, ranging from simple eddy-viscosity to differential Reynolds stress models. The DLR results presented at the fifth AIAA Drag Prediction Workshop have been obtained using standard eddy viscosity models, i. e. the Spalart-Allmaras (SA) model ${ }^{34}$ without the so-called $f_{t 1}$ and $f_{t 2}$ term (SA-noft) and the $\mathrm{k} \omega$-SST model by Menter in its 1994 version. ${ }^{35}$

Such linear eddy viscosity models cannot predict the anisotropy of turbulent normal stresses near walls which is considered responsible for secondary corner flow phenomena. Indeed, using the SA and the k $\omega$-SST model, a separation in the wing-fuselage intersection of the NASA CRM configuration is predicted at high incidence, which is not observed in the experiment. Improvement is expected by either non-linear extensions of eddy-viscosity models, e. g. explicit algebraic Reynolds stress models (EARSM), or full differential Reynolds stress models. In the following chapters the Quadratic Constitution Relation (QCR) extension in combination with the SA and the k $\omega$-SST models as well as the DLR Reynolds stress turbulence model are described.

\section{QCR-extension of eddy-viscosity models}

As has been shown in DPW by Yamamoto et al. and Sclafani et al., the corner flow separation can be suppressed, using the so-called Quadratic Constitution Relation (QCR) extension. ${ }^{36-38}$ This modification has been presented by Spalart ${ }^{39}$ as an example of how linear eddy viscosity models can be improved for predicting normal stress anisotropies. In general form it states the components of the Reynolds stress tensor as

$$
\bar{\rho} \widetilde{R}_{i j}=\bar{\rho} \widetilde{R}_{i j}^{(E V M)}+\bar{\rho} \widetilde{R}_{i j}^{(Q C R)}
$$

where $\bar{\rho} \widetilde{R}_{i j}^{(E V M)}$ are the Reynolds stress components according to any linear eddy viscosity model and

$$
\bar{\rho} \widetilde{R}_{i j}^{(Q C R)}=-c_{n l 1}\left[O_{i k}\left(\bar{\rho} \widetilde{R}_{j k}^{(E V M)}\right)+O_{j i k}\left(\bar{\rho} \widetilde{R}_{i k}^{(E V M)}\right)\right]
$$

is the QCR extension. The term

$$
O_{i j}=\frac{\frac{\partial \widetilde{U}_{i}}{\partial x_{j}}-\frac{\partial \widetilde{U}_{i}}{\partial x_{j}}}{\sqrt{\frac{\partial \widetilde{U}_{m}}{\partial x_{n}} \frac{\partial \widetilde{U}_{m}}{\partial x_{n}}}}
$$

denotes the components of the normalised rotation tensor with $\widetilde{U}_{i}$ representing the components of the mean velocity. The coefficient has been set to $c_{n l 1}=0.3$.

Note that the above formulation depends on the respective eddy viscosity model, since the Boussinesq hypothesis reads

$$
\bar{\rho} \widetilde{R}_{i j}^{(E V M)}=-2 \mu^{(t)} \widetilde{S}_{i j}^{*}+\frac{2}{3} \bar{\rho} \widetilde{k} \delta_{i j}
$$

where $\mu^{(t)}$ is the eddy viscosity,

$$
\begin{aligned}
\widetilde{S}_{i j} & =\frac{1}{2}\left(\frac{\partial \widetilde{U}_{i}}{\partial x_{j}}+\frac{\partial \widetilde{U}_{j}}{\partial x_{i}}\right) \\
\widetilde{S}_{i j}^{*} & =\widetilde{S}_{i j}-\frac{1}{3} \widetilde{S}_{k k} \delta_{i j}
\end{aligned}
$$

are the components of the simple and the traceless strain rate tensor and $\delta_{i j}$ is the Kronecker symbol. The crucial point is the specific kinetic turbulence energy $\widetilde{k}$, which is not available, e. g. with the SA model. For this reason the QCR extension (2) is cast into the following form

$$
\bar{\rho} \widetilde{R}_{i j}^{(Q C R)}=4 c_{n l 1} \mu^{(t)} \frac{\widetilde{\Omega}_{i k} \widetilde{S}_{j k}+\widetilde{\Omega}_{j k} \widetilde{S}_{i k}}{\sqrt{\widetilde{S}_{m n} \widetilde{S}_{m n}+\widetilde{\Omega}_{m n} \widetilde{\Omega}_{m n}}},
$$


which only contains the eddy viscosity $\mu^{(t)}$ and is independent of the availability of $\widetilde{k}$.

Furthermore, the production term of the $\widetilde{k}$-equation, employed e. g. by the k $\omega$-SST model, deserves attention, as it reads

$$
\bar{\rho} P^{(\widetilde{k})}=-\bar{\rho} \widetilde{R}_{k l} \widetilde{S}_{k l}=-\left(\bar{\rho} \widetilde{R}_{k l}^{(E V M)}+\bar{\rho} \widetilde{R}_{k l}^{(Q C R)}\right) \widetilde{S}_{k l}
$$

As can be shown,

$$
\bar{\rho} \widetilde{R}_{k l}^{(Q C R)} \widetilde{S}_{k l}=0
$$

so that the QCR extension does not alter the transport equations of the underlying eddy viscosity model. Thus, the QCR extension can be easily implemented in a general form by adding the corresponding fluxes $\partial / \partial x_{k}\left(\bar{\rho} \widetilde{R}_{i k}^{(Q C R)}\right)$ and $\partial / \partial x_{k}\left(\bar{\rho} \widetilde{R}_{i k}^{(Q C R)} \widetilde{U}_{i}\right)$ to the momentum and total energy equation, respectively.

The effect of the coefficient $c_{n l 1}$ on the predicted normal stress anisotropy can be analysed using standard assumptions on boundary layers. Assuming the wall-normal derivative of the wall parallel velocity component $\partial \widetilde{U} / \partial y$ being the only one non-zero component of the velocity gradient tensor, there are only two non-zero QCR stress components, i. e.

$$
\begin{aligned}
\bar{\rho} \widetilde{R}_{11}^{(Q C R)} & =2 c_{n l 1} \mu^{(t)} \frac{\partial \widetilde{U}}{\partial y}, \\
\bar{\rho} \widetilde{R}_{22}^{(Q C R)} & =-2 c_{n l 1} \mu^{(t)} \frac{\partial \widetilde{U}}{\partial y},
\end{aligned}
$$

where index 1 denotes the wall-parallel mean flow direction and index 2 the wall-normal direction. In the log-law

$$
\begin{aligned}
& \mu^{(t)}=\kappa \bar{\rho} u_{\tau} y, \\
& \frac{\partial \widetilde{U}}{\partial y}=\frac{u_{\tau}}{\kappa y},
\end{aligned}
$$

where $\kappa$ is the von Kármán constant and $u_{\tau}$ is the friction velocity. Furthermore, according to the Bradshaw hypothesis,

$$
\widetilde{k} u_{\tau}^{2}=\sqrt{c_{\mu}}=0.09
$$

so that one finally obtains the QCR stresses

$$
\begin{aligned}
& \bar{\rho} \widetilde{R}_{11}^{(Q C R)}=2 c_{n l 1} \widetilde{k}, \\
& \bar{\rho} \widetilde{R}_{22}^{(Q C R)}=-2 c_{n l 1} \widetilde{k} .
\end{aligned}
$$

Combination with the Boussinesq hypothesis (4) yields

$$
\begin{aligned}
\bar{\rho} \widetilde{R}_{11} & =2 \widetilde{k}\left(\frac{1}{3}+c_{n l 1} \sqrt{c_{\mu}}\right), \\
\bar{\rho} \widetilde{R}_{22} & =-2 \widetilde{k}\left(\frac{1}{3}+c_{n l 1} \sqrt{c_{\mu}}\right),
\end{aligned}
$$

from which the corresponding components of the Reynolds stress anisotropy tensor

$$
\widetilde{b}_{i j}=\frac{\widetilde{R}_{i j}}{2 \widetilde{k}}-\frac{1}{3} \delta_{i j}
$$

are directly obtained as

$$
\begin{array}{ll}
\widetilde{b}_{11}=c_{n l 1} \sqrt{c_{\mu}} & =0.09, \\
\widetilde{b}_{22}=-c_{n l 1} \sqrt{c_{\mu}} & =-0.09, \\
\widetilde{b}_{33} & =0 .
\end{array}
$$

As shown in Figure 10, computations for the flat plate, using the kw-SST model with QCR extension, confirm the above analysis. Note that for the flat plate neither the skin friction nor the velocity profile is affected by the QCR extension. In contrast a small upstream shift of the shock is found for the flow around the RAE 2822 airfoil, Case 9 and Case 10, ${ }^{40}$ confirming findings by Yamamoto et al. ${ }^{37}$ 


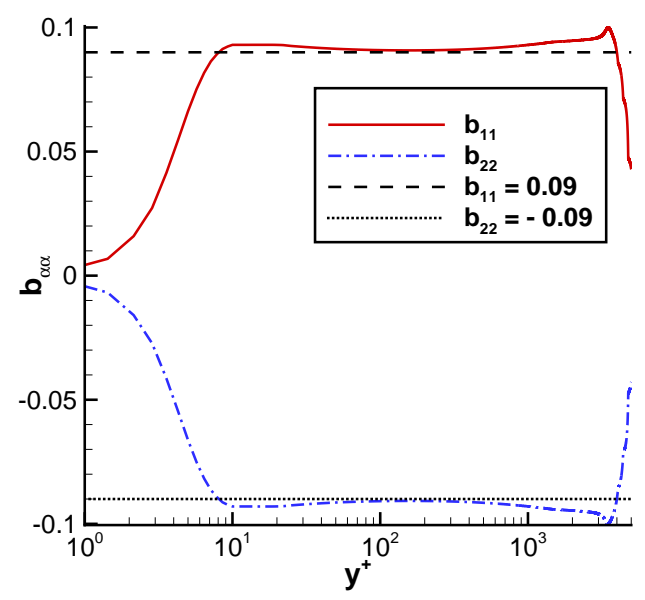

Figure 10. Flat plate, local Reynolds number $R_{x}=9.15 \cdot 10^{6}$. Reynolds stress anisotropy components due to QCR extension of the $\mathrm{k} \omega$-SST model.

\section{SSG/LRR- $\omega$ differential Reynolds stress model}

The SSG/LRR- $\omega$ model developed by $\mathrm{DLR}^{41}$ is based on the Reynolds stress transport equation for compressible flow

$$
\frac{\partial\left(\bar{\rho} \widetilde{R}_{i j}\right)}{\partial t}+\frac{\partial}{\partial x_{k}}\left(\bar{\rho} \widetilde{R}_{i j} \widetilde{U}_{k}\right)=\bar{\rho} P_{i j}+\bar{\rho} \Pi_{i j}-\bar{\rho} \epsilon_{i j}+\bar{\rho} D_{i j}+\bar{\rho} M_{i j},
$$

where the production term is exactly given by

$$
\bar{\rho} P_{i j}=-\widetilde{R}_{i k} \frac{\partial \widetilde{U}_{j}}{\partial x_{k}}-\widetilde{R}_{j k} \frac{\partial \widetilde{U}_{i}}{\partial x_{k}}
$$

The Re-distribution term is modeled as

$$
\begin{aligned}
& \bar{\rho} \Pi_{i j}=-\left(C_{1} \bar{\rho} \epsilon+\frac{1}{2} C_{1}^{*,(S S G / L R R)} \bar{\rho} P_{k k}\right) \widetilde{b}_{i j} \\
& +C_{2}^{(S S G / L R R)} \bar{\rho} \epsilon\left(\widetilde{b}_{i k} \widetilde{b}_{k j}-\frac{1}{3} \widetilde{b}_{m n} \widetilde{b}_{m n} \delta_{i j}\right)+\left(C_{3}^{(S S G / L R R)}-C_{3}^{*,(S S G / L R R)} \sqrt{\widetilde{b}_{m n} \widetilde{b}_{m n}}\right) \bar{\rho} \widetilde{k} \widetilde{S}_{i j}^{*} \\
& +C_{4}^{(S S G / L R R)} \bar{\rho} \widetilde{k}\left(\widetilde{b}_{i k} \widetilde{S}_{j k}+\widetilde{b}_{j k} \widetilde{S}_{i k}-\frac{2}{3} \widetilde{b}_{m n} \widetilde{S}_{m n} \delta_{i j}\right)+C_{5}^{(S S G / L R R)} \bar{\rho} \widetilde{k}\left(\widetilde{b}_{i k} \widetilde{W}_{j k}+\widetilde{b}_{j k} \widetilde{W}_{i k}\right),
\end{aligned}
$$

where the dissipation rate $\epsilon$ is computed from the specific dissipation rate $\omega$ according to

$$
\epsilon=C_{\mu} \widetilde{k} \omega
$$

with $C_{\mu}=0.09$ and the specific kinetic turbulence energy is related to the trace of the specific Reynolds stress tensor by

$$
\widetilde{k}=\frac{1}{2} \widetilde{R}_{i i}
$$

The coefficient values vary from the LRR-values near the wall to the SSG-values at the outer edge of the boundary layer according to

$$
\begin{aligned}
C_{i}^{(S S G / L R R)} & =F_{1} \widehat{C}_{i}^{(L R R)}+\left(1-F_{1}\right) \widehat{C}_{i}^{(S S G)} \\
C_{i}^{*,(S S G / L R R)} & =\left(1-F_{1}\right) \widehat{C}_{i}^{*,(S S G)}
\end{aligned}
$$

where $F_{1}$ is Menter's blending function. ${ }^{35}$ The bounding values are listed in table 2. Note that the value of the LRR-parameter $C_{2}^{(L R R)}=0.52$ has been recently modified. ${ }^{42}$ 
Table 2. Values of closure coefficients for the SSG and the LRR contributions to the SSG/LRR- $\omega$ re-distribution term. $C_{2}^{(L R R)}=0.52$.

\begin{tabular}{cccccccc} 
& $\widehat{C}_{1}$ & $\widehat{C}_{1}^{*}$ & $\widehat{C}_{2}$ & $\widehat{C}_{3}$ & $\widehat{C}_{3}^{*}$ & $\widehat{C}_{4}$ & $\widehat{C}_{5}$ \\
\hline SSG & 3.4 & 1.8 & 4.2 & 0.8 & 1.3 & 1.25 & 0.4 \\
& & & & & & $\frac{18 C_{2}^{(L R R)}+12}{11}$ & $\frac{-14 C_{2}^{(L R R)}+20}{11}$
\end{tabular}

The dissipation term is modeled as anisotropic tensor with components

$$
\bar{\rho} \epsilon_{i j}=\frac{2}{3} \epsilon \delta_{i j}
$$

Different from, ${ }^{41}$ the diffusion term is modeled here by simple gradient diffusion

$$
\bar{\rho} D_{i j}=\frac{\partial}{\partial x_{k}}\left[\left(\bar{\mu}+D^{(S S G / L R R)} \frac{\bar{\rho} \widetilde{k}}{\omega}\right) \frac{\partial \widetilde{R}_{i j}}{\partial x_{k}}\right]
$$

for enhancing numerical robustness. In this, $\bar{\mu}$ is the averaged molecular viscosity, and the diffusion coefficient varies between the bounding LRR and SSG values according to

$$
D^{(S S G / L R R)}=F_{1} \sigma^{*}+\left(1-F_{1}\right) \frac{2}{3} \frac{C_{s}}{C_{\mu}}
$$

with $\sigma^{*}=0.5$ and $C_{s}=0.22$.

The specific dissipation rate is provided by Menter's baseline $\omega$-equation, ${ }^{35}$ where $\omega$ is limited similar to Durbin's suggestion for eddy-viscosity models. ${ }^{43}$

According to Wilcox, ${ }^{44}$ in the LRR part the following components of the anisotropy tensor, are obtained

$$
\begin{gathered}
\widetilde{b}_{11}=\frac{8+12 \widehat{C}_{2}^{(L R R)}}{33 \widehat{C}_{1}^{(L R R)}} \approx 0.120 \\
\widetilde{b}_{22}=\frac{2-30 \widehat{C}_{2}^{(L R R)}}{33 \widehat{C}_{1}^{(L R R)}} \approx-0.114 \\
\widetilde{b}_{33}=\frac{18 \widehat{C}_{2}^{(L R R)}-10}{33 \widehat{C}_{1}^{(L R R)}} \approx-0.005,
\end{gathered}
$$

where the little difference to zero trace of the numerical values is due to round-off errors. As one can see, the absolute values are larger than those associated with the QCR-extension, and in particular $\widetilde{b}_{33} \neq 0$.

\section{Computational Grids}

\section{A. CFD Grids}

\section{Common Grids}

A six level common grids family of point-matched O-O topology multi-block grids has been build by Boeing. ${ }^{45}$ The sequence is based on an extra-fine grid (L5) with $40.9 \cdot 10^{6}$ hexahedral elements. To limit grid sizes a 2-to-3 cell grid generation strategy was applied. The L6 grid (ultra fine) has been generated by refining L5 by 1.5 in each parameter direction. The coarser grids L4-L2 and L3-L1 have been defined by dividing the grids by 8 appropriate for multigrid. ${ }^{45}$ The derived medium grid (L3) with $5.1 \cdot 10^{6}$ elements represents a current grid size in industry for wing-fuselage configurations and will be used for the fluid-structure-coupled calculations and is presented in figure 11 . 


\section{DLR Custom Grids}

Since DPW-3 DLR is investigating unstructured prismatic and hexahedral elements dominated grids regarding their capabilities to be used for accurate drag predictions for aircraft ${ }^{11,12}$ with the software packages Centaur ${ }^{\mathrm{TM}} 46$ and Solar. $^{47}$ The hexahedral based approach offers potentially higher stretched elements, e.g. in wing span wise direction, because discretization errors are larger for prismatic elements when highly stretched. In DPW-5 it was one objective of DLR to further compare the grid convergence behaviour of the common grids with a hexahedral grid family generated with Solar.

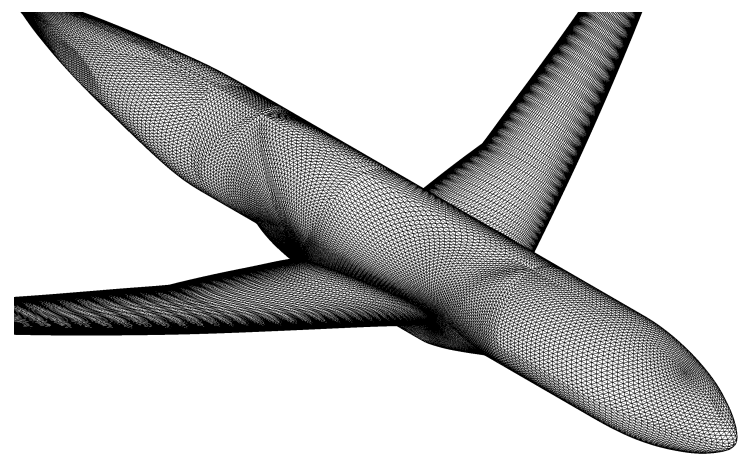

Figure 11. Common hexahedral grid of DPW-5.

Because of the difficulty of adequate element shapes and sizes in corners with the Solar hex-dominated technique, additionally three levels of Solar plus an overlapping full H-topology hexahedral grid block have been generated too. Due to the fact that Centaur ${ }^{\mathrm{TM}}$ is a very mature software, that prisms dominated grids are well established at the institute, and because Centaur ${ }^{\mathrm{TM}}$ offers hexahedral elements near the walls and partly in the field another objective was to compare the results of all four grid types (common hexahedral, Solar, Solar plus overlapping hex-block, Centaur ${ }^{\mathrm{TM}}$ ) with/without hexahedral wake blocks for Case 2. Results have been published in the frame of the the German Aerodynamics Workshop STAB. ${ }^{13}$ Figure 12 shows the Solar, Solar plus overlapping, and Centaur ${ }^{\text {TM }}$ grids. Here only the common grids L3, L4, and the Centaur grids with hexahedral wake block are applied. Grid sizes as well as the turbulence models used with these grids are listed in table 3 .

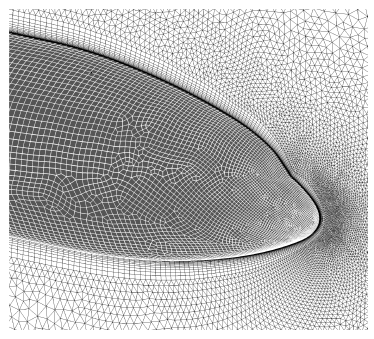

(a) Solar

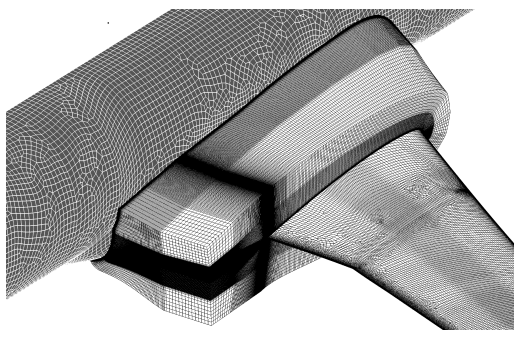

(b) Solar + hexahedral overlapping block

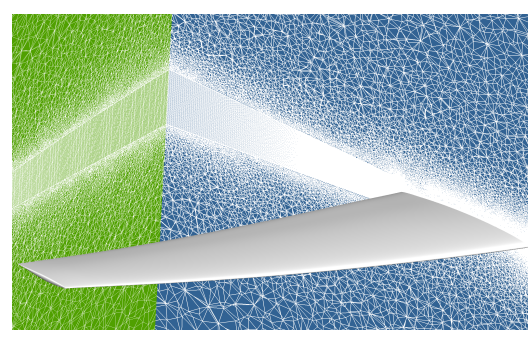

(c) Centaur

Figure 12. Overview of DLR custom grids applied in DPW-5.

Table 3. Grids and turbulence models applied for case 2, CFD grid nodes in million.

\begin{tabular}{|c|c|c|c|c|}
\hline \multirow[t]{2}{*}{ Level } & \multicolumn{2}{|l|}{ Common } & \multicolumn{2}{|c|}{$\begin{array}{c}\text { Centaur } \\
+ \text { hexa-wake }\end{array}$} \\
\hline & Turb & Nodes & Turb & Nodes \\
\hline 3 & $\mathrm{SA}, \mathrm{SA}+\mathrm{QCR}, k \omega-\mathrm{SST}, k \omega-\mathrm{SST}+\mathrm{QCR}, \mathrm{SSG} / \mathrm{LLR}-\omega$ & 5.2 & SA, SA-QCR & 37.4 \\
\hline 4 & $\mathrm{SA}, \mathrm{SA}+\mathrm{QCR}, k \omega-\mathrm{SST}, k \omega-\mathrm{SST}+\mathrm{QCR}, \mathrm{SSG} / \mathrm{LLR}-\omega$ & 17.4 & & \\
\hline
\end{tabular}

\section{B. CSM Model}

A NASTRAN ${ }^{\circledR}$ finite-element structural model of wing, fuselage, horizontal tail plane, engine nacelles, and balance interface was made available by NASA Langley, figure 13. The model includes both right and left sides to account for the wind tunnel model's non-symmetric inner structure. Joints between individual 
components are modeled with rigid body elements. A rigid suspension is assumed at the balance interference. The finite-element discretization consists of $1.37 \cdot 10^{6}$ nodes, $6.82 \cdot 10^{6}$ elements, and $20.46 \cdot 10^{6}$ degrees-offreedom. For the coupled simulations the engine nacelles and pylons were removed to more accurately represent the actual wind tunnel configuration. Coupling of aerodynamic loads between CFD simulation and finite-element analysis is established on the wing upper and lower surfaces.

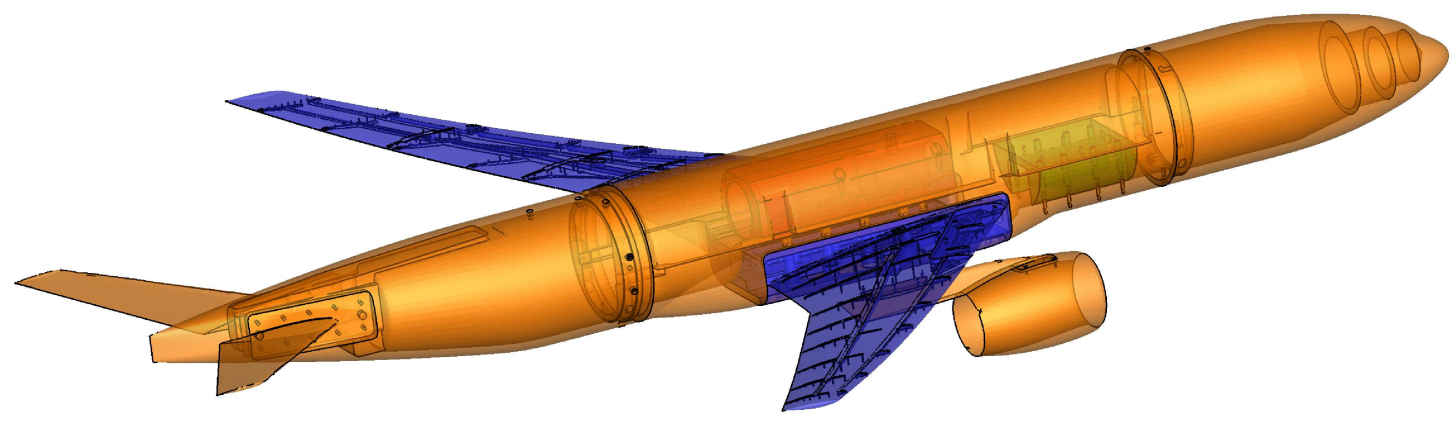

Figure 13. CRM finite-element model.

\section{Results}

\section{A. FSC Simulations}

The purpose of the fluid-structure-coupled simulations here is to determine the static aeroelastic equilibrium state for one selected DPW-5 test case and to assess the influence of wing deformations on static pressure distributions and overall aerodynamic coefficients, in particular the pitching moment. This will help to quantify the effects, which have caused the observed deviations between CFD simulations and experiments.

FSC simulations were run for DPW-5 test case 2 (cf. Chapter A) using the medium (L3) common grid and SA turbulence model. For an improved evaluation along with results obtained during recent investigations of support system effects, ${ }^{20,21}$ the angle-of-attack is varied from 0.0 to $4.0^{\circ}$.

Generally, the aeroelastic effects observed on the CRM were found to be larger than for the DLR-F6 wing-body configuration ${ }^{32}$ used during the Second and Third Drag Prediction Workshops. In figure 14 , the overall aerodynamic coefficients $C_{L}$ and $C_{D}$ obtained from the conventional CFD and FSC simulations, respectively, together with experimental data from the NTF wind tunnel test campaign (Test 197, Run 44), are plotted as a function of angle-of-attack. The coupled simulation results show lower overall lift coefficient values compared to the conventional CFD results, with the difference between FSC and CFD increasing with angle-of-attack. The lift reduction is due to the nose-down wing twist deformation induced by the geometric bending-torsion-coupling of the backward-swept wing as the wing is bent upwards by the external aerodynamic loads.

The drop in lift starting between $\alpha=3.0^{\circ}$ and $\alpha=3.5^{\circ}$ found in the numerical data is due to an over-prediction of the side-of-body flow separation size in the medium (L3) hexahedral grid when using the SA turbulence model. The separation effect and related numerical issues were investigated and discussed previously in section $\mathrm{B}$. In the linear region, i.e. for $\alpha \leq 3.0^{\circ}$, deviations between the coupled simulation results and experimental data are considerably smaller than for the conventional CFD analysis, table 4. The remaining error is very similar to the differences found by Rivers et al. ${ }^{20,21}$ to be caused by the model support system. This suggests that including both aeroelastic and support system effects in the numerical simulation, together with a physically correct turbulence model, will allow for removing most of the previously observed deviations.

Differences in drag coefficient between conventional CFD and FSC remain very small for incidence angles up to $2.5^{\circ}$. The deviation at off-design conditions is caused by the shock-induced flow separation on the outboard wing. In the coupled simulation, the separation starts to develop later, i.e. at a higher angle-ofattack, and, compared to the conventional CFD analysis, extends over a smaller spanwise portion of the outboard wing. This is due to the lower local angles-of-attack in the outer region of the deformed wing.

For pitching moment coefficients, figure 15, the deviations between numerical and experimental data 


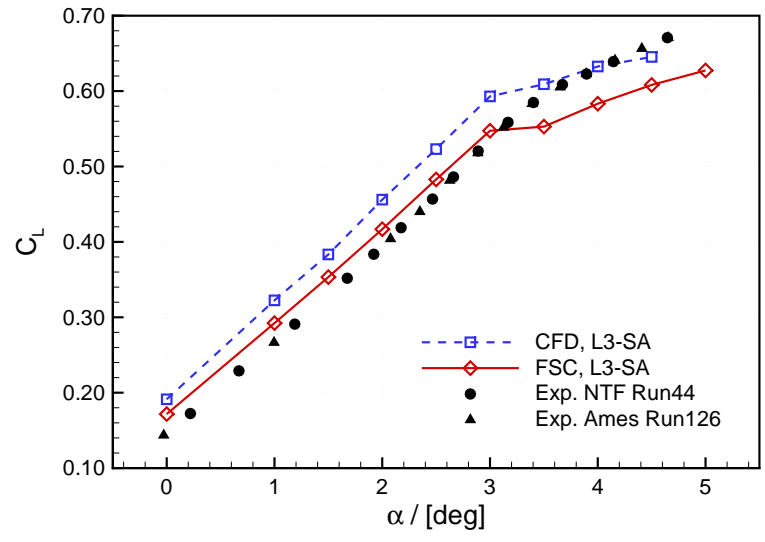

(a) Lift

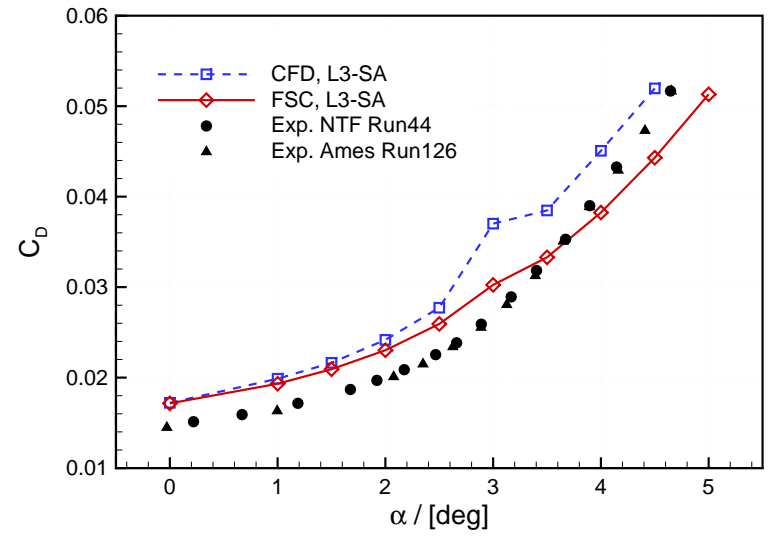

(b) Drag

Figure 14. Overall aerodynamic lift and drag coefficients for DPW-5 test case 2.

Table 4. Lift coefficient deviations between numerical predictions and experimental results.

\begin{tabular}{ccc}
\hline$\alpha$ & $\Delta C_{L, C F D}$ & $\Delta C_{L, F S C}$ \\
\hline $0.0^{\circ}$ & 0.0456 & 0.0260 \\
$3.0^{\circ}$ & 0.0574 & 0.0118 \\
\hline
\end{tabular}

is greatly reduced by taking into account wing deformation in the coupled simulation. Still, considerable differences remain, even around the design point. Again, including support system effects appears likely to move the numerical predictions closer to the experimental data.

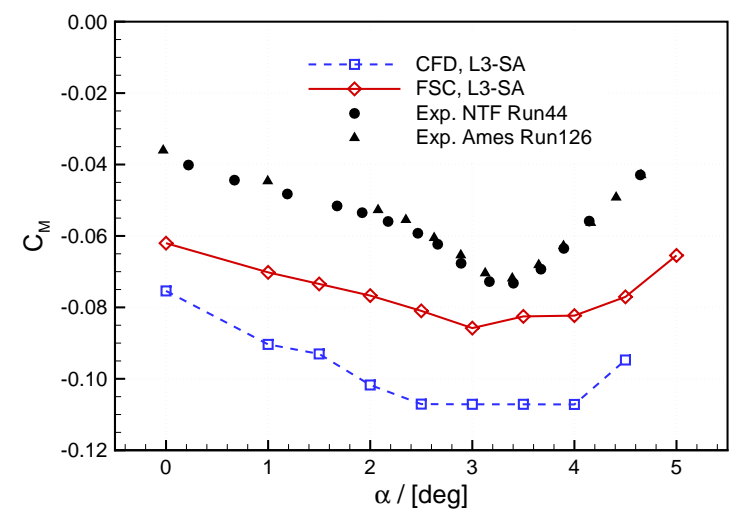

Figure 15. Pitching moment coefficient for DPW-5 test case 2.

Figure 16 shows a comparison of chordwise static pressure distributions between CFD and FSC simulations and wind tunnel test data taken from the NTF campaign for four different spanwise wing sections at $\alpha=3.0^{\circ}$. At the innermost section, figure 16 (a), where wing deformations are very small, cf. figure 17 (b), both numerical methods are in good agreement with each other and the measured pressure distribution. Although twist deformation in this section is only about $-0.0115^{\circ}$, the shock location predicted by the FSC simulation lies somewhat closer to the wind tunnel data. The mid-wing section, figure 16 (b), already shows some effects of aero-elastic deformation between the leading edge and about $75 \%$ chord, with a decreased rooftop pressure level, reduced pressure along most of the wing lower side, and, again, a more precise shock location. At $\eta=-0.727$, figure 16 (c), wing twist has increased to $\varepsilon=-1.09^{\circ}$ and the differences between conventional CFD and FSC become even more apparent. Here, only the coupled simulation is in good agreement with both measured rooftop pressure levels and shock location. At the outermost section, figure 16 (d), 
the shock location as predicted by the FSC simulation has moved significantly downstream and a doubleshock pattern has developed. Twist deformation has increased to $\varepsilon=-1.41^{\circ}$ considerably reducing the local incidence angle. As a result, the pressure distribution at $\eta=-0.950$ resembles those for conventional CFD computations at lower angles-of-attack, cf. figures 2 (b) and 3 (b), where a similar double-shock system exists. Unfortunately, the true shock position can not be determined from the experimental data due to an insufficient spatial resolution of pressure taps.

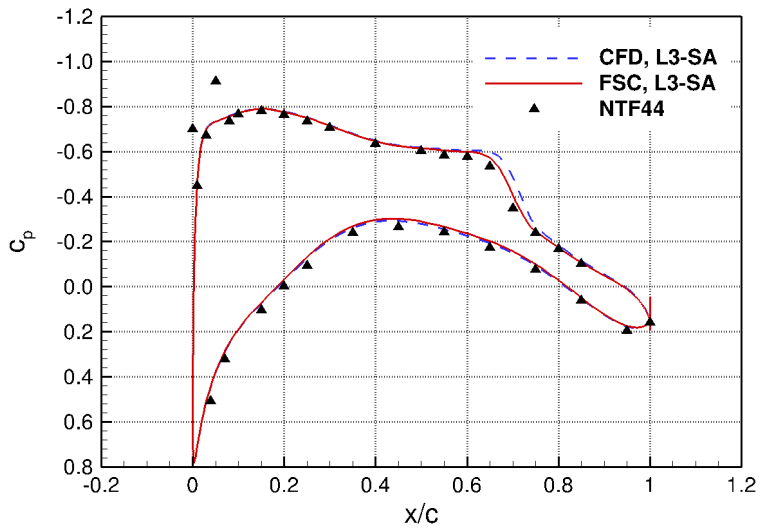

(a) $\eta=0.131$

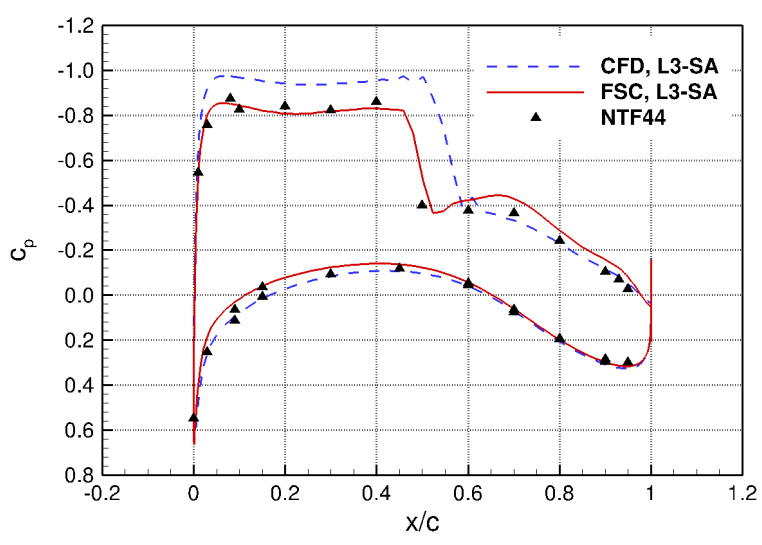

(c) $\eta=0.727$

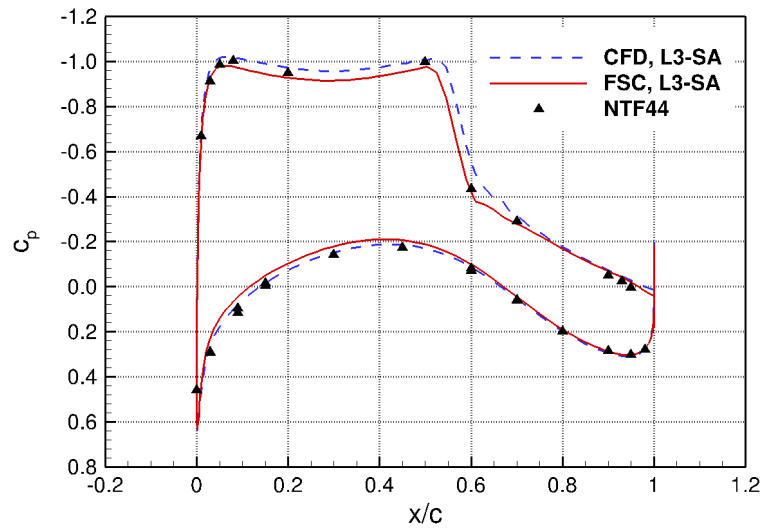

(b) $\eta=0.502$

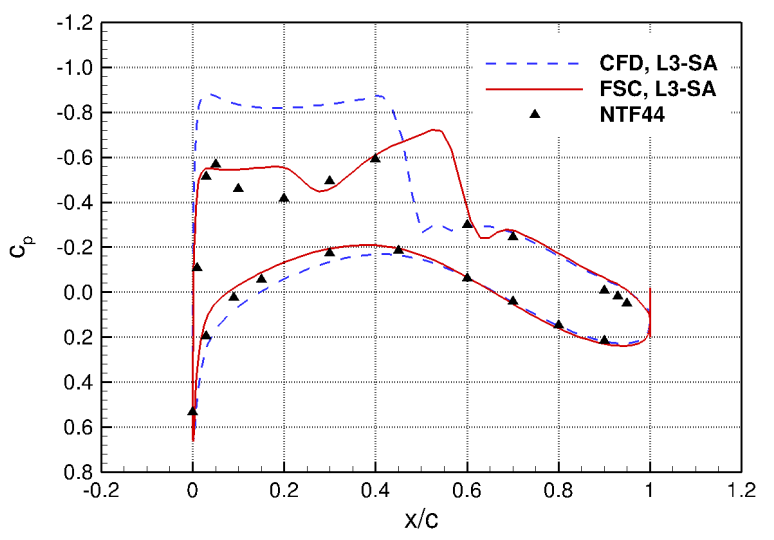

(d) $\eta=0.950$

Figure 16. Chordwise static pressure distribution at $\alpha=3.0^{\circ}$ for different spanwise wing sections; TAU only (CFD), fluid-structure-coupled (FSC), and NASA NTF experiments (NTF).

In figure 17 the wing bending and twist deformations are plotted as a function of angle-of-attack at wing tip (a) and as spanwise distribution for $\alpha=3.0^{\circ}$ (b). As previously seen with lift coefficient, figure 14 (a), good linearity exists for $\alpha \leq 3.0^{\circ}$. Between $\alpha=3.0^{\circ}$ and $\alpha=3.5^{\circ}$ the onset of the side-of-body flow separation can be identified by small decreases in both bending and twist deformations. The declining slope for $\alpha>3.0^{\circ}$ is caused by the growing shock-induced flow separation on the outer wing.

The spanwise wing twist and bending distribution for $\alpha=3.0^{\circ}$ is plotted in figure 17 (b). Due to the fact that from $\eta \approx 0.40$ outward the $\mathrm{c} / 4$-line lies behind the model reference center, any aeroelastic wing deformation will not only result in a change of spanwise lift distribution, but also reduce the overall nosedown pitching moment. Unfortunately, no experimental deformation data was available for comparison as the corresponding wind tunnel test was still ongoing at the time of publication of this paper.

\section{B. Turbulence model study}

In this section the influence of turbulence models on the prediction of side-of-body flow separation for the CRM wing-body configuration is investigated. Here two eddy viscosity models, i.e the one-equation model by Spalart-Allmaras (SA) and the shear stress transport (kw-SST) $k-\omega$ two-equation model by Menter, and the differential Reynolds stress model (SSG/LRR- $\omega$ ) developed at DLR are applied. For this study the 


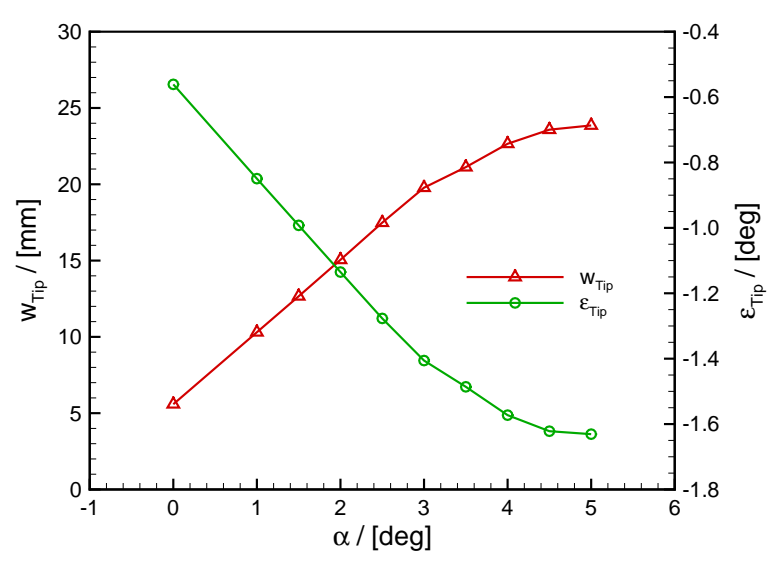

(a) Wing tip deformation

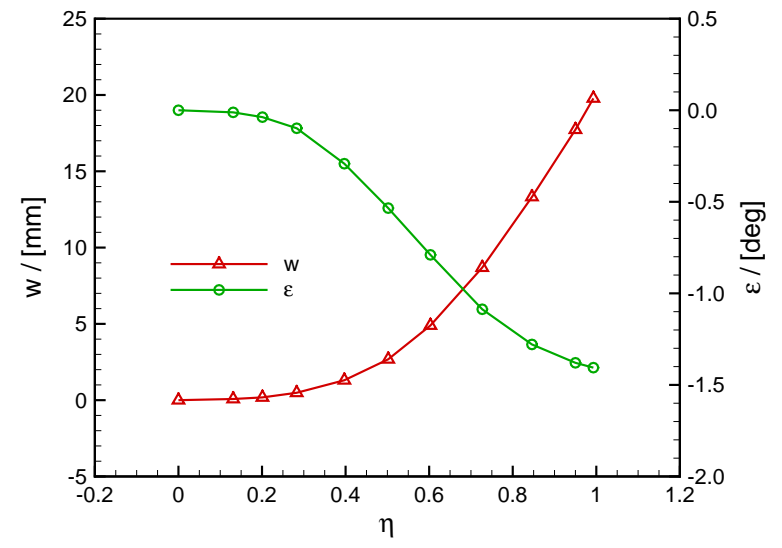

(b) Spanwise wing deformation

Figure 17. Wing Bending and Twist Deformations.

CRM hybrid grids of medium level (L3) with 5 million nodes and of fine level (L4) with 17 million nodes have been used. On the medium grid, computations are performed with the SA, SA with QCR extension (SA+QCR); $k \omega$-SST, $k \omega$-SST with QCR extension (SST+QCR); and the SSG/LRR- $\omega$ models, whereas the predictions on the fine grid are obtained by the SA, SA+QCR and the SSG/LRR- $\omega$ models only. In addition the SA and SA+QCR models are also applied in combination with the Centaur grid to verify the effects on a prismatic element dominated grid too.

In this study, the viscous fluxes of main and turbulent equations are discretized using central differences. The inviscid fluxes of the main equations are calculated by a central scheme with matrix dissipation. For the turbulent equations the convective fluxes are approximated with a central scheme for the SA model and with second-order Roe's scheme for the $\mathrm{k} \omega$-SST and SSG/LRR- $\omega$ turbulence models. Steady computations are performed using a semi - implicit lower-upper symmetric Gauss-Seidel (LU-SGS) method. For the computations here, slightly different numerical dissipation parameters than typical TAU dissipation settings have been used. The parameters used here lead to slightly more dissipation. This is done in-order to be able to obtain a steady converged solution with all three turbulence models on the grids employed.

Simulations were performed for the CRM wing-body configurations from $\alpha=2.5^{\circ}$ to $\alpha=4.0^{\circ}$ and for selected cases up to $\alpha=4.25^{\circ}$ with incidence angle increment of $\Delta \alpha=0.25^{\circ}$. For the cruise design condition $(\mathrm{CL}=0.5)$ simulation is carried out using the solution of $\alpha=2.5^{\circ}$.

In figure 18, the force and moment coefficients predicted by different turbulence models on the L3 grid are shown. In the figure experimental data are used for reference purposes. With all the turbulence models continuous increase in lift as the incidence angle increased is predicted - no lift breakdown is observed. The SA model predicted higher lift at all the incidence angles than the k $\omega$-SST and SSG/LRR- $\omega$ models. Below the incidence angle of $3.5^{\circ}$ the $\mathrm{k} \omega$-SST model predicted lower lift than SSG/LRR- $\omega$. For $\alpha>3.5^{\circ}$ the $\mathrm{k} \omega$-SST and SSG/LRR- $\omega$ delivered almost the same lift coefficient. The eddy viscosity models with QCR extension delivered lower lift compared to the corresponding non-QCR models.

The drag force polar demonstrates that the SA model delivers lower drag than the k $\omega$-SST and SSG/LRR$\omega$ models and the drag predicted by the latter model is in between the predictions delivered by the $\mathrm{k} \omega$-SST and SA models. With QCR extension both the SA and $\mathrm{k} \omega$-SST models predict higher drag than the corresponding models without QCR extension.

The pitching moment coefficient is displayed in figure 18(c) for different turbulence models. The SA model predicts the lowest pitching moment coefficient compared to the $\mathrm{k} \omega$-SST and SSG/LRR- $\omega$ models. The predictions delivered by SSG/LLR- $\omega$ can be found in between the predictions of the SA and k $\omega$-SST. The eddy viscosity models with QCR extension predicted a higher pitching moment coefficient compared to non-QCR models.

In figure 19 the $C p$ distribution predicted on the L3 grid at $\alpha=3^{\circ}$ and $\alpha=4^{\circ}$ is compared for different turbulence models at two spanwise sections of the wing. Here the experimental data are used as reference. Differences persist in the location of the shock and in the pressure distribution in the separated flow region. At $\alpha=3^{\circ}$ the SA model predicts the location of the shock slightly downstream of the SSG/LRR- $\omega$ predictions. 


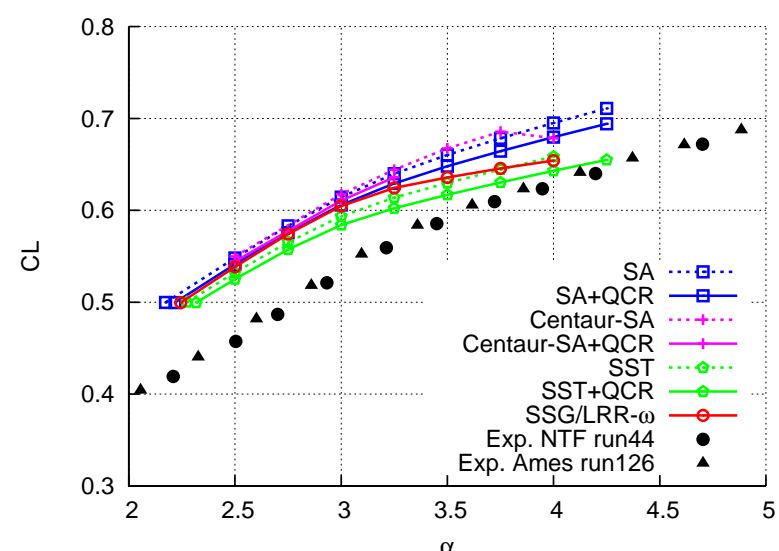

(a) Lift

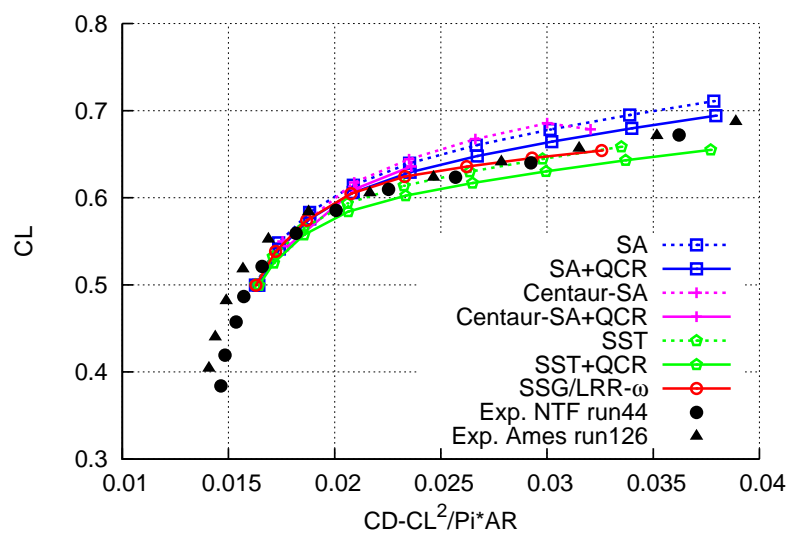

(b) Idealized drag

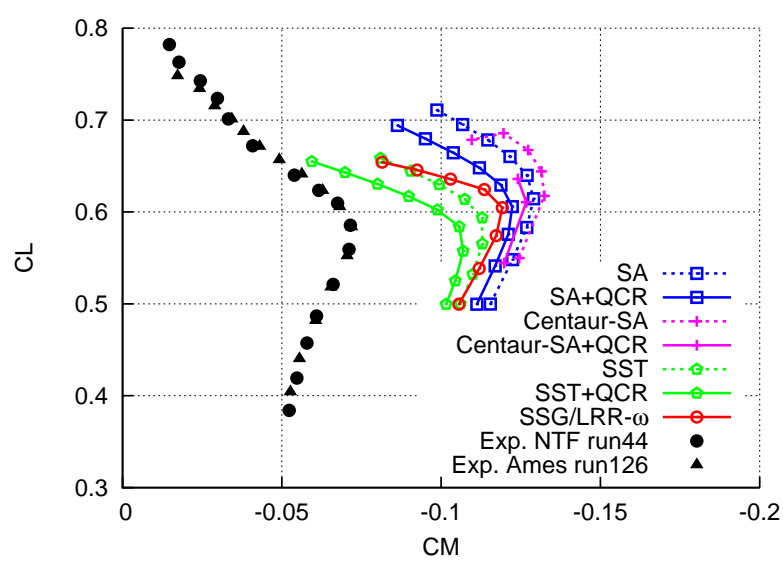

(c) Pitching moment

Figure 18. Comparison of force and pitching moment coefficients for different turbulence models on L3 and Centaur grid.

The k $\omega$-SST model predictions unveil that the shock is predicted slightly upstream of the shock location delivered by the SSG/LRR- $\omega$. The upstream shift of the shock by the k $\omega$-SST model is about half of the difference between the SA and SSG/LRR- $\omega$ models. At $\alpha=4^{\circ}$ the SA model predicts lower pressure on the suction side of wing than the other turbulence models and delivers the shock slightly downstream of the $\mathrm{k} \omega$-SST and SSG/LRR- $\omega$ predictions. At this incidence angle very small difference between the k $\omega$-SST and $\mathrm{SSG} / \mathrm{LRR}-\omega$ with regard to shock location is observed.

With QCR extension the SA and k $\omega$-SST models shift the shock location slightly upstream compared to the corresponding non-QCR models. This upstream movement of the shock caused larger shock induced separation on the main and outboard of the wing which lead to lower lift and higher pitching moment (see figure $18(\mathrm{c}))$.

To demonstrate the influence of grid refinement on the predictions, drag and moment coefficients predicted on L3 and L4 grids are shown in figure 20 for the SA, SA+QCR and SSG/LRR- $\omega$ models. The predictions obtained by the SSG/LRR- $\omega$ unveil grid independence up to the incidence angle of $3.5^{\circ}$. On the L4 grid the SA model with and without QCR predicted slightly higher lift and lower pitching moment coefficients than on L3 grid.

In figure $21 C p$ distributions predicted on the two different grids by the SA+QCR and SSG/LRR- $\omega$ models at $\alpha=3^{\circ}$ are shown here. The SA model predictions are not depicted as the difference between predictions on both grids is of same magnitude that was observed with SA+QCR and hence only the SA+QCR results are discussed. For the SSG/LRR- $\omega$ Reynolds stress model no significant influence of grid refinement was observed, whereas with the other model downstream movement of shock was predicted on the fine mesh. This is the possible reason for slightly higher lift and lower pitching moment coefficients on the L4 mesh by the SA and SA+QCR models (see figure 20). Please note that no fluid-structure coupling is applied for the 


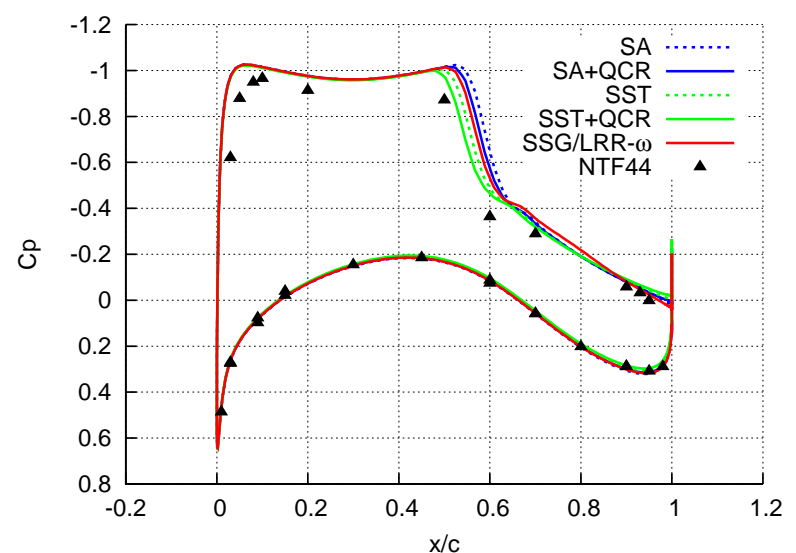

(a) $\eta=0.5\left(\stackrel{\mathrm{x} / \mathrm{c}}{\alpha=3^{\circ}}\right)$

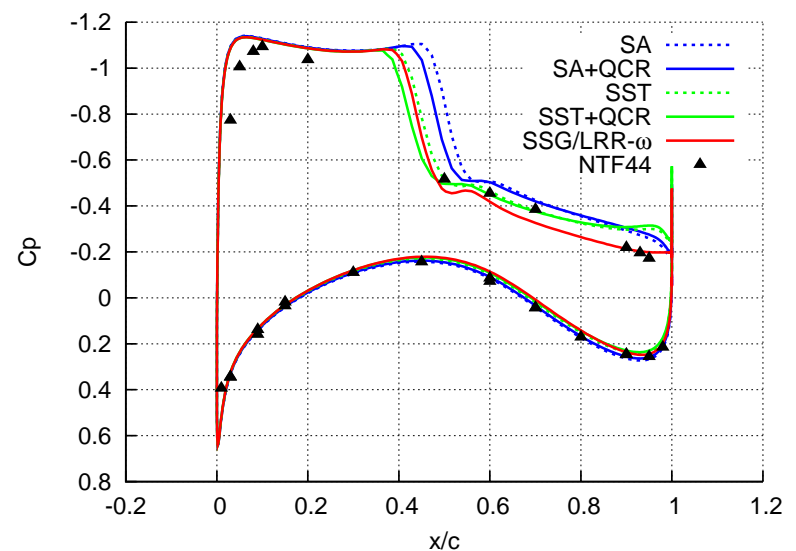

(c) $\eta=0.50\left(\alpha=4^{\circ}\right)$

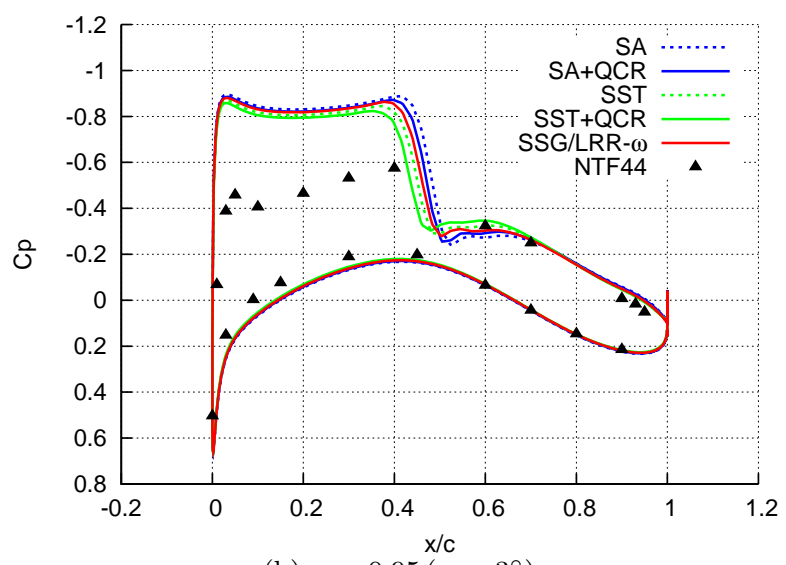

(b) $\eta=0.95\left(\alpha=3^{\circ}\right)$

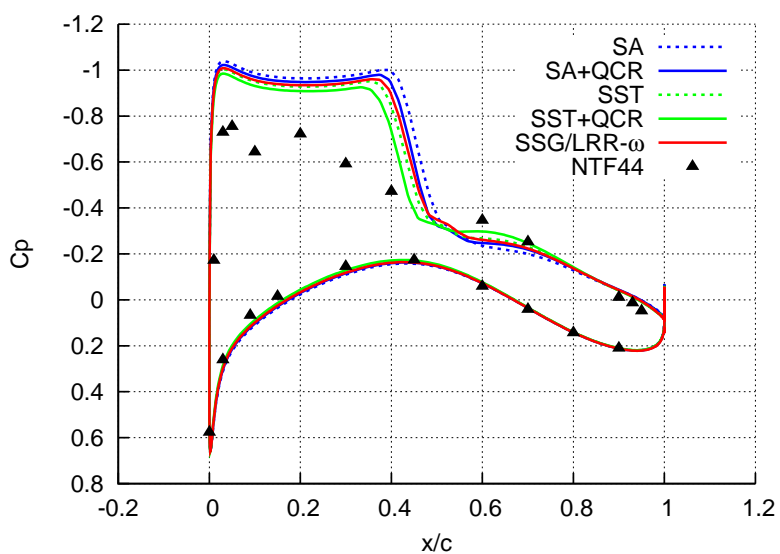

(d) $\eta=0.95\left(\alpha=4^{\circ}\right)$

Figure 19. $C p$ distribution at spanwise sections of the wing for different turbulence models on L3 grid at $\alpha=3^{\circ}$ and $\alpha=4^{\circ}$.

turbulence models investigation. As observed in DPW a large discrepancy of experimental and numerical data exists at $\eta=0.95$ due to reasons investigated by Rivers et al. ${ }^{20,21}$

To examine the influence of turbulence models on the side-of-body flow separation surface skin friction lines for the $\alpha=3^{\circ}$ case are displayed in figure. 22. On the L3 grid a very small separation is predicted with the SA model whereas with the SA+QCR, $\mathrm{k} \omega$-SST, $\mathrm{k} \omega$-SST+QCR and the SSG/LRR- $\omega$ models no separation in the side-of-body region is predicted. On the Centaur grid a sightly larger side.of.body separation can be observed for the SA model due to the significant higher grid resolution in that area compared to the L2 grid. Applying the SA+QCR model again a reduction of the size of the separation is achieved. As the incidence angle increases, the SA model predictions show an increased size of side-of-body flow separation. However, the size of flow separation is not as big as it is predicted by Yamamoto et al. ${ }^{37}$ and Sclafani et al. ${ }^{38}$ on the same hybrid L3 grid. The reason for this small separation is marginally higher dissipation which in the current investigation arose from slightly different numerical dissipation parameters that were different from typical TAU settings but that had been necessary to be consistent for all grids. Despite small separation in the side-of-body region comparisons are made for different turbulence models. Surface skin friction lines obtained at $\alpha=4^{\circ}$ by different turbulence models are shown in figure 23. The SA model predicted large separation whereas the $\mathrm{k} \omega$-SST and SSG/LRR- $\omega$ predicted no separation in the region. With QCR extension the side-of-body flow separation is reduced by the SA model. The k $\omega$-SST+QCR model predicted a similar flow topology as the $\mathrm{k} \omega$-SST predictions.

Predictions obtained on the L4 grid by the SA, SA+QCR and SSG/LRR- $\omega$ models are depicted in figure 24. The SA model predicted larger flow separation on the L4 grid than on the L3 grid at $\alpha=3^{\circ}$. At higher incidence angle much larger separation is predicted by the SA model. This separation extent is reduced by the QCR extension (see figures 25). The SSG/LRR- $\omega$ has not predicted any side-of-body 


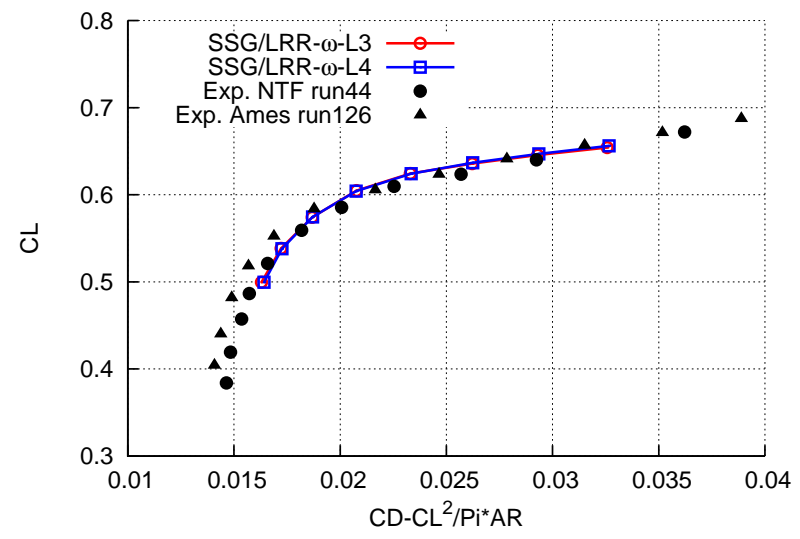

(a) Idealized drag (SSG/LRR- $\omega)$

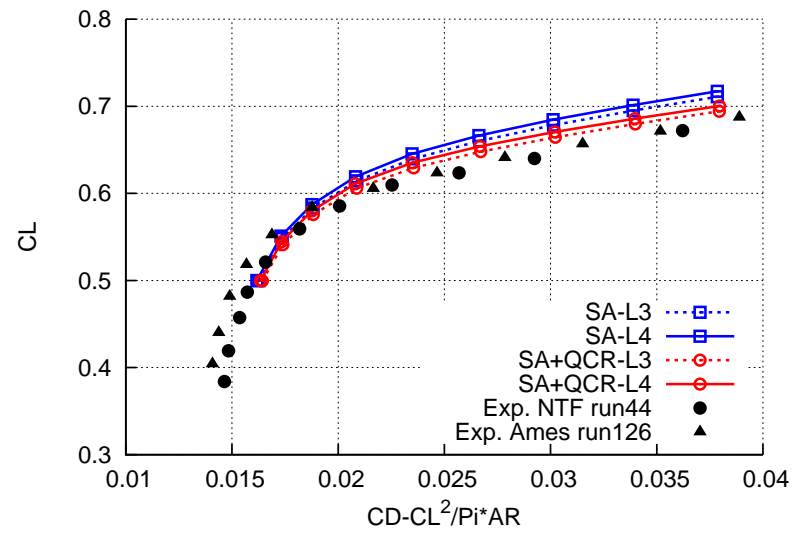

(c) Idealized drag (SA)

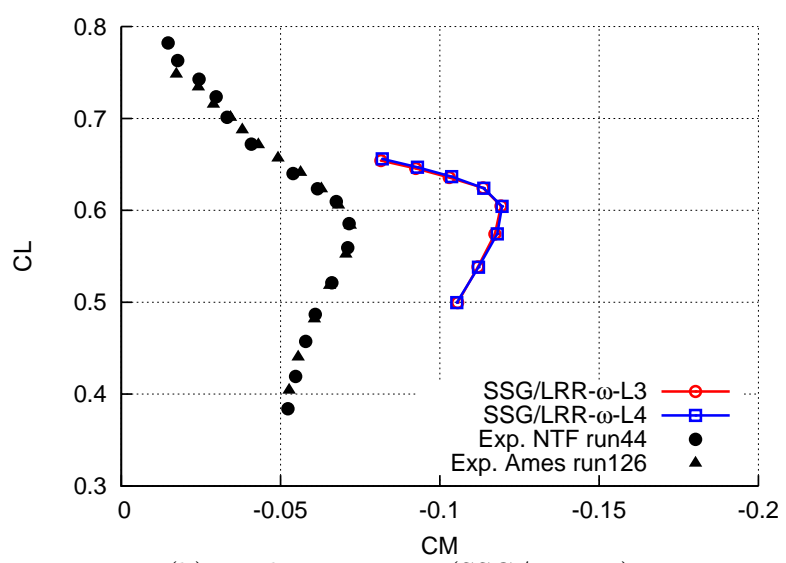

(b) Pitching moment (SSG/LRR- $\omega$ )

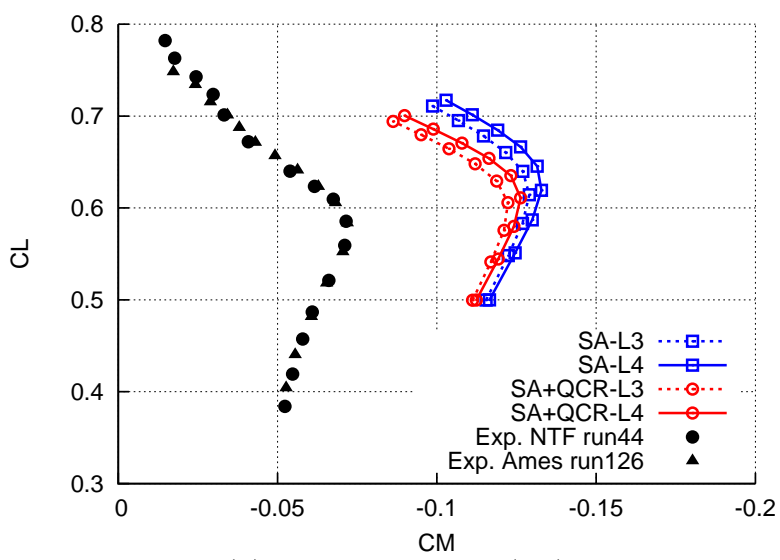

(d) Pitching moment (SA)

Figure 20. Comparison of force and pitching moment coefficients for the SA and SSG/LRR- $\omega$ models on L3 and L4 grids.

separation on the grid. To illustrate the size of the separation in the wing-body junction in comparison with the wing size a detailed view of the wing with skin friction lines is displayed in figure 26.

In the experiments of the CRM no separation in the side-of-body region was observed. Such trend is reproduced with the SSG/LRR- $\omega$. With QCR extension, which accounts for the anisotropy of normal Reynolds stresses, the separation extent is reduced but it is not completely eliminated in the present investigation.

\section{Conclusions}

the influence of wing deformations on static pressure distributions and overall aerodynamic coefficients of NASA's Common Research Model were investigated using the medium (L3) common grid, the SA turbulence model, and a finite-element structural model kindly provided by NASA Langley. Static fluid-structurecoupled simulations were run at $M_{\infty}=0.85$ and $R e=5 \cdot 10^{6}$ with the angle-of-attack varying between 0.0 and $4.0^{\circ}$. Numerical results were compared to experimental data from a wind tunnel test campaign in NASA's National Transonic Facility. Generally, the deviations of lift, drag, and pitching moment coefficients observed between DPW-4 and DPW-5 computational results and measured data are considerably reduced by taking into account elastic wing deformations. Lift coefficient values predicted by the coupled simulation are lower than for the conventional CFD computations, leading to considerably smaller deviations from the experimental data. For drag coefficients, significant differences between the conventional CFD and FSC analyses only occur at off-design flow conditions and are mostly due to variations in the development of the shock-induced separation on the outboard wing. Deviations between numerical and experimental pitching moment coefficients are substantially reduced by taking into account wing deformation. Due to 


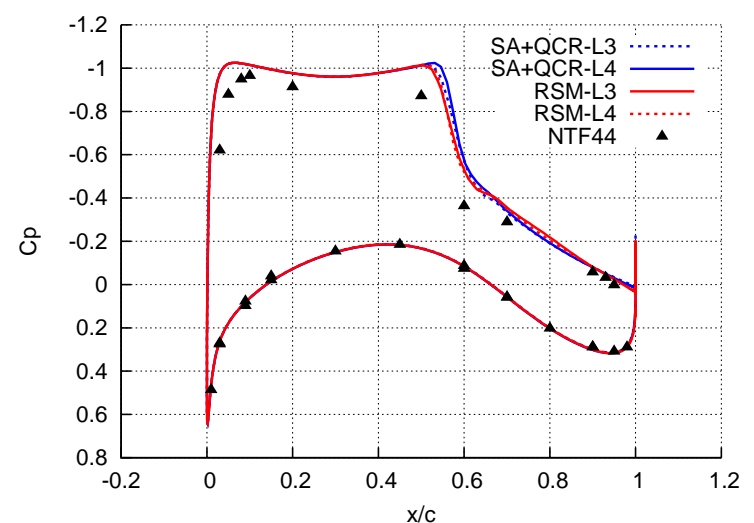

(a) $\eta=0.50\left(\alpha=3^{\circ}\right)$

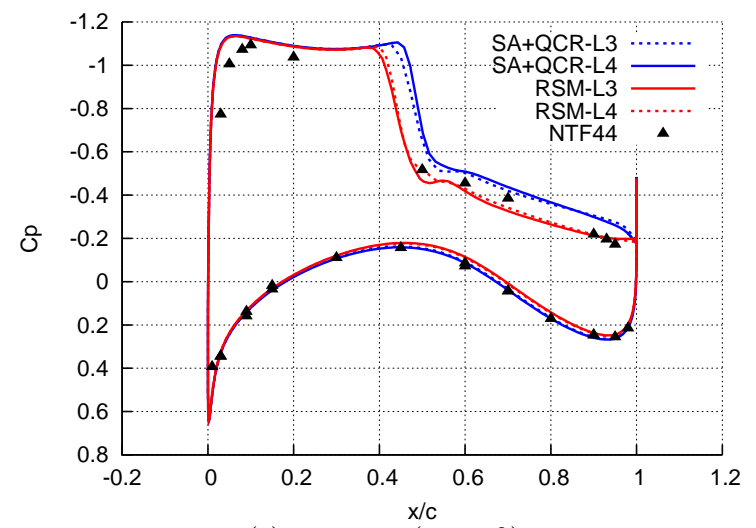

(c) $\eta=0.50\left(\alpha=4^{\circ}\right)$

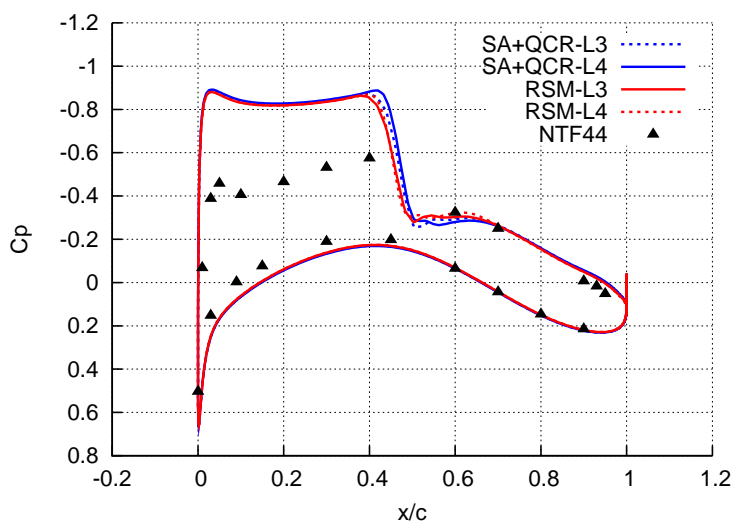

(b) $\eta=0.95\left(\alpha=3^{\circ}\right)$

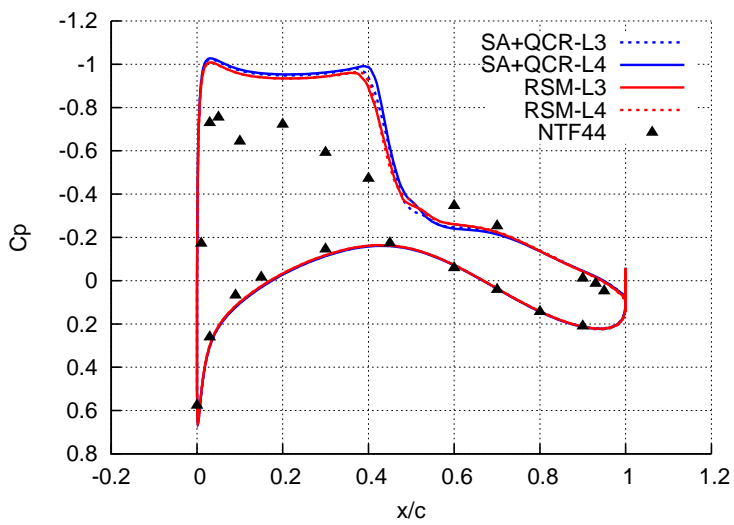

(d) $\eta=0.95\left(\alpha=4^{\circ}\right)$

Figure 21. $C p$ distribution at spanwise sections of the wing for different turbulence models on different grids at $\alpha=3^{\circ}$ and $\alpha=4^{\circ}$.

the pitching moment's strong sensitivity with respect to the overall static pressure distribution, differences remain relatively large. Regarding chordwise static pressure distributions, some minor aeroelastic effects become visible in the mid-wing section, increasing in magnitude towards the wing tip. In general, the FSC simulations provide a significantly more accurate prediction of rooftop pressure levels, pressure distribution on the wing lower side, and shock location. Wing bending and twist deformations show a good linearity for $\alpha \leq 3.0^{\circ}$. For higher angles-of-attack, wing deformations are influenced by the side-of-body flow separation and the shock-induced flow separation on the outer wing. Based on the results found in this study and by Rivers et al. ${ }^{20,21}$ it is suggested that further numerical investigations should include both aeroelastic and support system effects, together with a high-quality turbulence model.

In a second part, a turbulence model study was performed for the CRM wing-body configuration on medium (L3) and fine (L4) hybrid grids provided by DPW-5 committee. Here eddy viscosity models, i.e the SA and kw-SST models, with and without QCR extension and the SSG/LRR- $\omega$ differential Reynolds stress model were applied. On the L3 grid the k $\omega$-SST model predicted lower lift and higher pitching moment coefficient than the SA and SSG/LRR- $\omega$ models. The aerodynamic coefficients delivered by the SSG/LRR- $\omega$ were in between the prediction of the SA and $\mathrm{k} \omega$-SST models. With QCR extension, the SA and k $\omega$-SST predicted the shock slightly upstream compared to the corresponding non-QCR models and hence larger shock induced separation on the main and outboard of the wing, which lead to lower lift and higher pitching momentum coefficients. The flow separation in the side-of-body region predicted by the SA model was very small at cruise design conditions which increased in size as the incidence angle increased. On the L4 grid much larger separation was predicted than on the L3 grid at the corresponding incidence angle. The QCR extension in combination with the SA model only reduced size of separation but it did not eradicate side-ofbody flow separation. The force, pitching moment coefficients and the surface pressure distribution predicted by the SA model with and without QCR extension were found to be grid depended. On the L4 grid slightly 


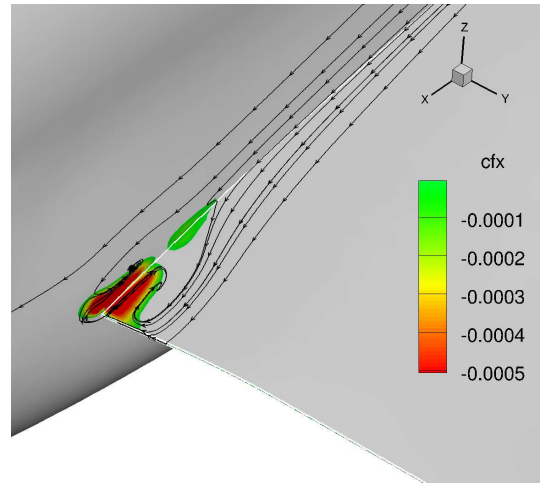

(a) SA (Centaur)

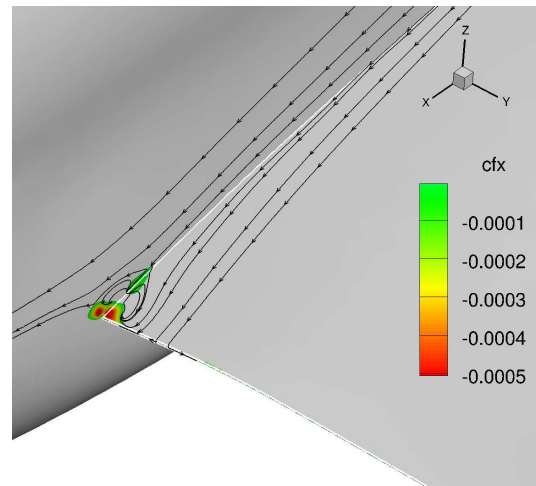

(d) $\mathrm{SA}+\mathrm{QCR}$ (Centaur)

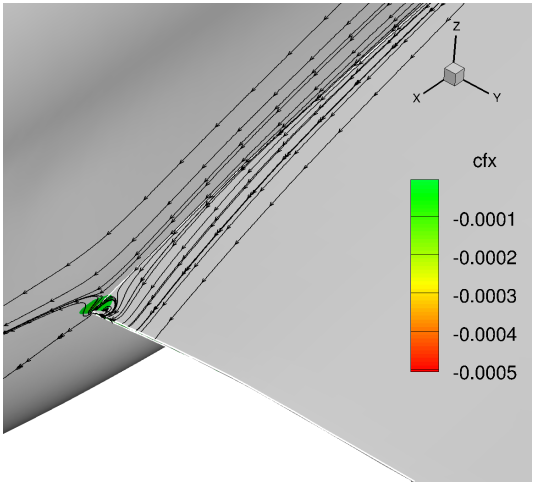

(b) SA (L3)

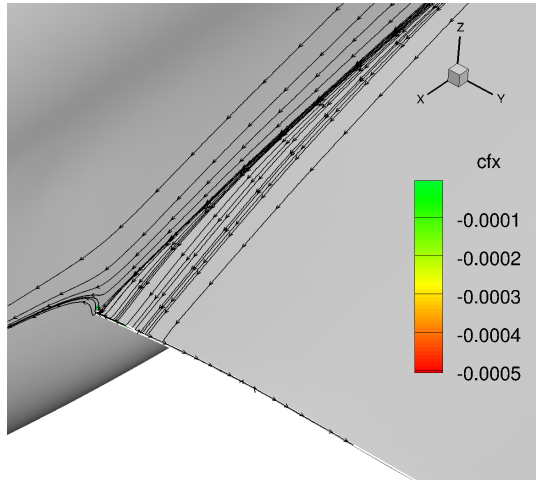

(e) $\mathrm{SA}+\mathrm{QCR}(\mathrm{L} 3)$

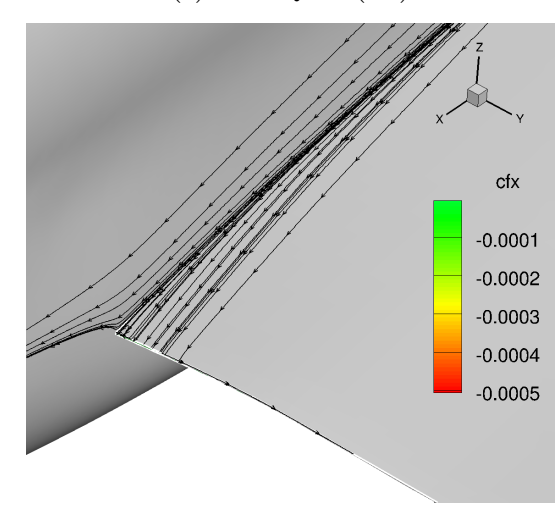

(g) RSM (L3)

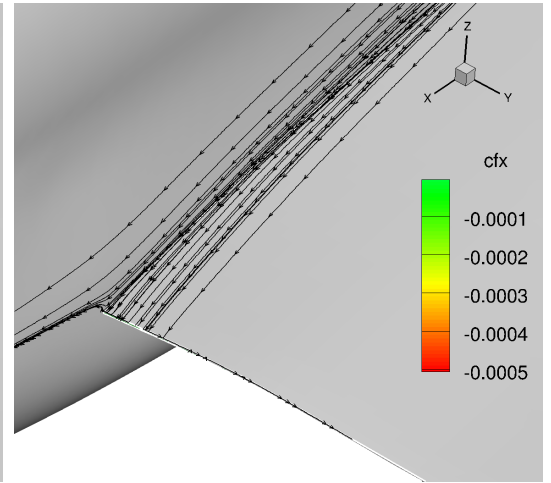

(c) $\operatorname{SST}(\mathrm{L} 3)$

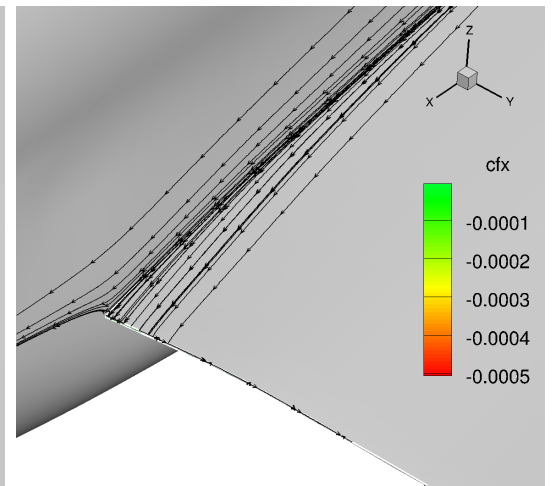

(f) $\mathrm{SST}+\mathrm{QCR}$ (L3)

(1)

Figure 22. Comparison of side-of-body separation at $\alpha=3^{\circ}$ for different turbulence models on L3 and Centaur grids. 


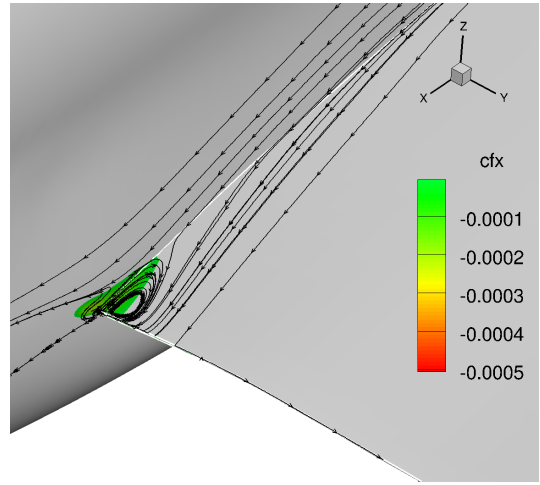

(a) SA

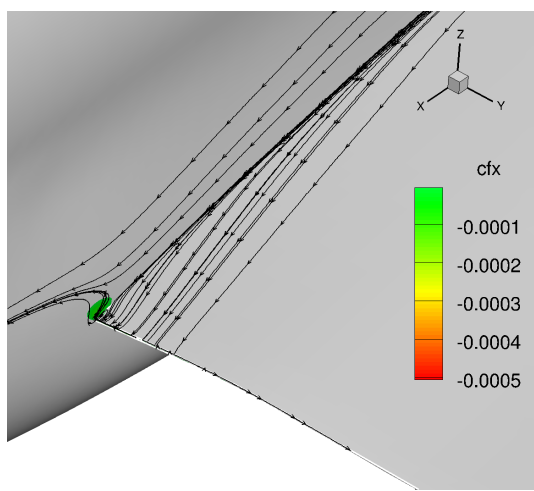

(c) $\mathrm{SA}+\mathrm{QCR}$

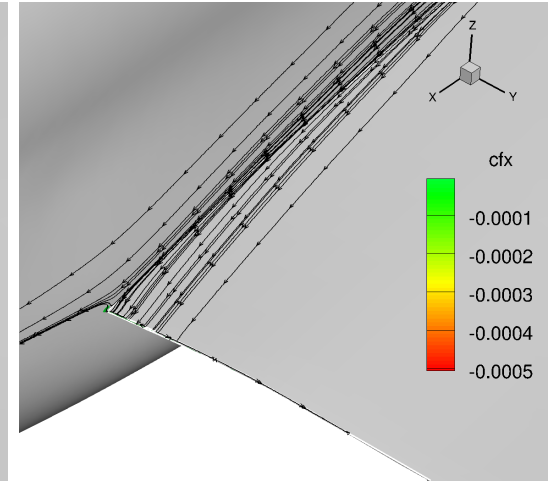

(b) $\mathrm{SST}$

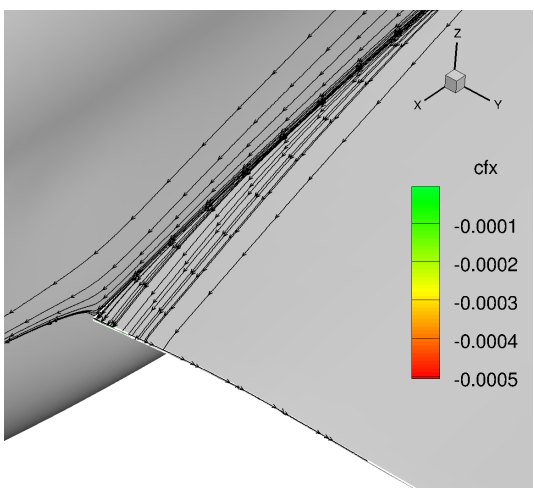

(d) $\mathrm{SST}+\mathrm{QCR}$

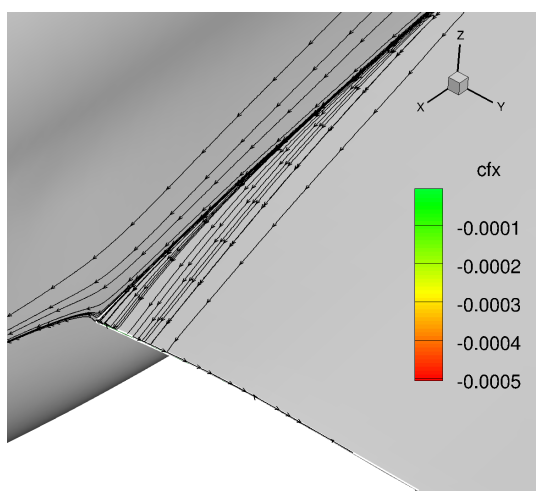

(e) RSM

Figure 23. Comparison of side-of-body separation at $\alpha=4^{\circ}$ for different turbulence models on L3 grid. 


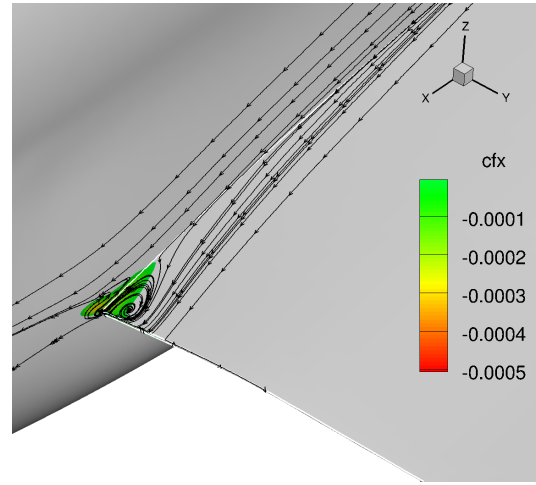

(a) SA

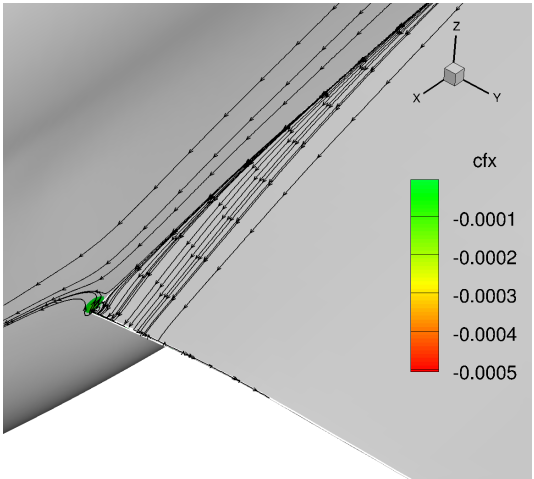

(b) $\mathrm{SA}+\mathrm{QCR}$

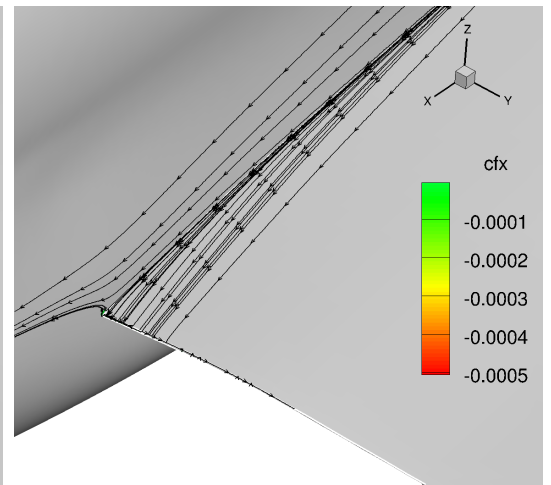

(c) RSM

Figure 24. Comparison of side-of-body separation at $\alpha=3^{\circ}$ for different turbulence models on L4 grid.

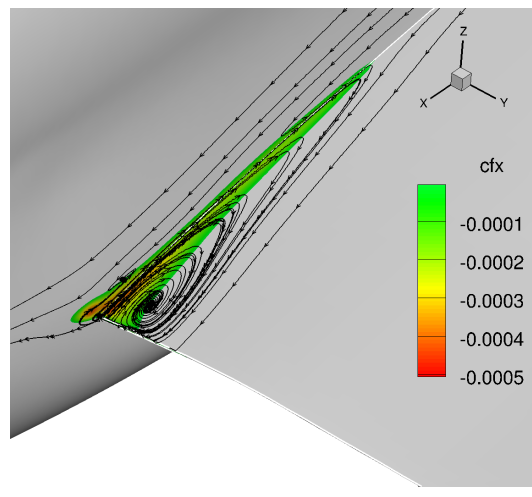

(a) SA

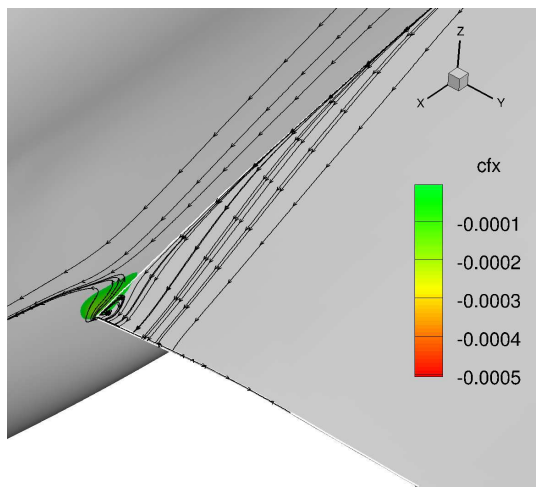

(b) SA+QCR

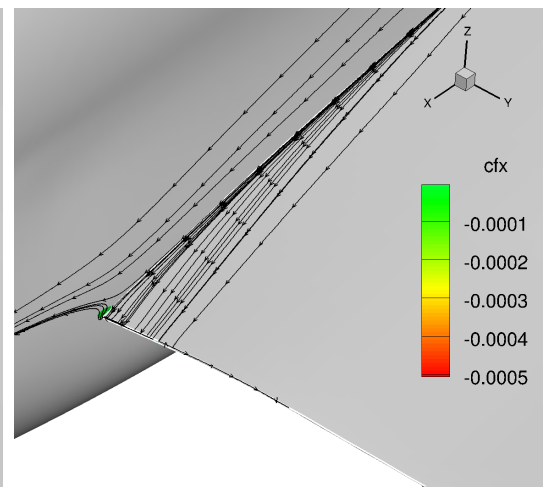

(c) RSM

Figure 25. Comparison of side-of-body separation at $\alpha=4^{\circ}$ for different turbulence models on L4 grid. 


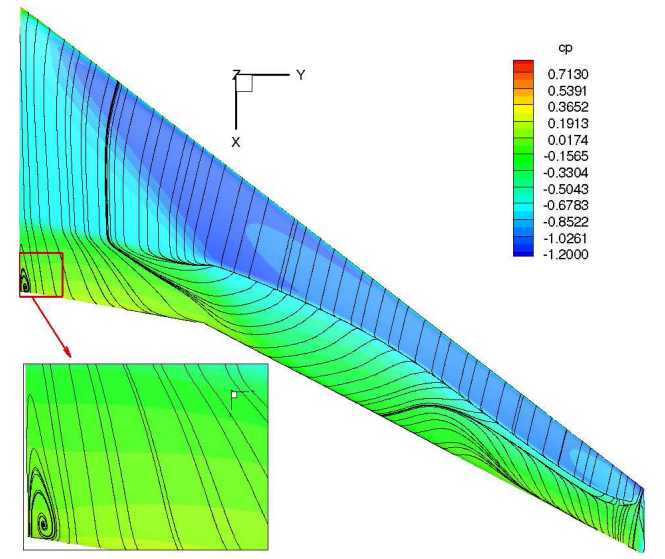

(a) SA

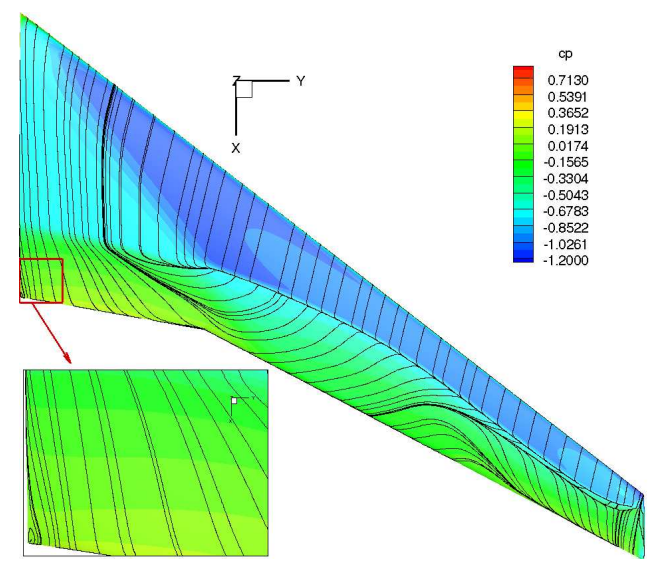

(b) $\mathrm{SA}+\mathrm{QCR}$

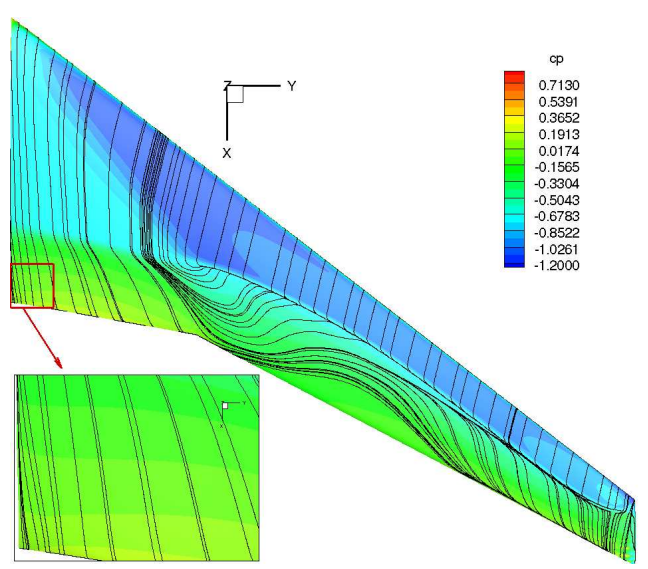

(c) SSG/LRR- $\omega$

Figure 26. Comparison of skin friction lines on the wing at $\alpha=4.0^{\circ}$ for different turbulence models on L4 grid. 
higher lift, lower drag and moment coefficients were predicted and the shock was located slightly downstream compared to the predictions on L3 grid. With the k $\omega$-SST model both with and without QCR extension side-of-body flow separation was not observed on the L3 grid. The lift, drag, pitching moment coefficients and the surface pressure distribution predicted by the SSG/LRR- $\omega$ model on both grids were observed to be grid independent. On both grids the SSG/LRR- $\omega$ predicted attached flow in side-of-body region which is in agreement with the experimental observations. The results on the DLR custom Centaur grid showed a slightly larger side-of-body separation can be computed with the SA model due to the higher grid resolution compared to the L3 and L4 grids. The application of the SA+QCR model reduces the separation, similar as shown on the common grids.

\section{Acknowledgments}

The authors would like to thank the current AIAA DPW Committee members, namely in alphabetical order of their organisations: J. Vassberg (Boeing), E. Tinoco (Boeing), M. Mani (Boeing), B. Rider (Boeing), D. Levy (Cessna), K. Laflin (Cessna), T. Zickuhr (Cessna), M. Murayama (JAXA), R. Wahls (NASA), J. Morrison (NASA), D. Mavriplis (University of Wyoming) for the excellent collaboration.

\section{References}

${ }^{1}$ Becker, K. and Vassberg, J., "Numerical Aerodynamics in Transport Aircraft Design," Notes on Numerical Fluid Mechanics and Multidisciplinary Design, edited by E.-H. Hirschel and E. Krause, Vol. 100, Springer, 2009, pp. 209-220.

${ }^{2}$ Rossow, C.-C. and Cambier, L., "European Numerical Aerodynamics Simulation Systems," Notes on Numerical Fluid Mechanics and Multidisciplinary Design, edited by E.-H. Hirschel and E. Krause, Vol. 100, Springer, 2009, pp. 189-208.

${ }^{3}$ Levy, D., Zickuhr, T., Vassberg, J., Agrawal, S., Wahls, R., Pirzadeh, S., and Hemsch, M., "Summary of Data from the First AIAA CFD Drag Prediction Workshop," AIAA Paper 2002-0841, Jan. 2002

${ }^{4}$ Laflin, K., Klausmeyer, S., Zickuhr, T., Vassberg, J., Wahls, R., Morrison, J., Brodersen, O., Rakowitz, M., Tinoco, E., and Godard, J.-L., "Data Summary from Second AIAA Computational Fluid Dynamics Drag Prediction Workshop." AIAA Journal of Aircraft, Vol. 42, No. 5, 2005, pp. 1165-1178.

${ }^{5}$ Vassberg, J., Tinoco, E., Mani, M., Brodersen, O., Eisfeld, B., Wahls, R., Morrison, J., Zickuhr, T., Laflin, K., and Mavriplis, D., "Abridged Summary of the Third AIAA Computational Fluid Dynamics Drag Prediction Workshop," AIAA Journal of Aircraft, Vol. 45, No. 3, pp. 781-798, 2008.

${ }^{6}$ Vassberg, J., Tinoco, E., Mani, M., Zickuhr, T., Levy, D., Brodersen, O., Crippa, S., Wahls, R., Morrison, J., Mavriplis, D., and Murayama, M., "Summary of the Fourth AIAA Drag Prediction Workshop." Paper 2010-4547, AIAA, June 2010.

${ }^{7}$ Levy, D., Laflin, K., Tinoco, E., Vassberg, J., Mani, M., Rider, B., Rumsey, C., Wahls, R., Morrison, J., Brodersen, O., Crippa, S., Mavriplis, D., and Murayama, M., "Summary of Data from the Fifth AIAA CFD Drag Prediction Workshop," AIAA Paper to be published, Jan. 2013.

${ }^{8}$ AIAA, "Drag Prediction Workshop," [online database], http://aaac.larc.nasa.gov/tsab/cfdlarc/aiaa-dpw, 2013.

${ }^{9}$ Rakowitz, M., Sutcliffe, M., Eisfeld, B., Schwamborn, D., Bleeke, H., and Fassbender, J., "Structured and Unstructured Computations on the DLR-F4 Wing-Body Configuration," Paper 2002-0837, AIAA, 2002.

${ }^{10}$ Brodersen, O., Rakowitz, M., Amant, S., Larrieu, P., Destarac, D., and Sutcliffe, M., "Airbus, ONERA, and DLR Results from the Second AIAA Drag Prediction Workshop." AIAA Journal of Aircraft, Vol. 42, No. 4, pp. 932-940, 2005.

${ }^{11}$ Brodersen, O., Eisfeld, B., Raddatz, J., and Frohnapfel, P., "DLR Results from the Third AIAA CFD Drag Prediction Workshop." AIAA Journal of Aircraft, Vol. 45, No. 3, pp. 823-836, 2008.

${ }^{12}$ Brodersen, O., Crippa, S., Eisfeld, B., Keye, S., and Geisbauer, S., "DLR Results from the Fourth AIAA CFD Drag Prediction Workshop," AIAA Paper 2010-4223, June 2010.

${ }^{13}$ Brodersen, O. and Crippa, S., "RANS-based Aerodynamic Drag and Pitching Moment Predictions for the Common Research Model." to be publsihed, DGLR STAB Workshop 2012.

${ }^{14}$ Rossow, C.-C., Godard, J., Hoheisel, H., and Schmitt, V., "Investigation of Propulsion Integration Interference on a Transport Aircraft Configuration," AIAA Paper 92-3097, June 1992.

${ }^{15}$ Rudnik, R., Sitzmann, M., Godard, J.-L., and Lebrun, F., "Experimental Investigation of the Wing-Body Juncture Flow on the DLR-F6 Configuration in the ONERA S2MA Facility," Paper 2009-4113, AIAA, 2009.

${ }^{16}$ Vassberg, J., DeHaan, M., Rivers, S., and Wahls, R., "Development of a Common Research Model for Applied CFD Validation Studies," Paper 2008-6919, AIAA, June 2008.

${ }^{17}$ Rivers, M., "Experimental Investigations on the NASA Common Research Model," Paper 2010-4218, AIAA, June 2010.

${ }^{18}$ Rivers, M. and Dittberner, A., "Experimental Investigations of the NASA Common Research Model in the NASA Langley National Transonic Facility and NASA Ames 11-Ft Transonic Wind Tunnel," Paper 2011-1126, AIAA, January 2011.

${ }^{19}$ Zilliac, G., Pulliam, T., Rivers, M., Zerr, J., Delgado, M., Halcomb, N., and Lee, H., "A Comparison of the Measured and Computed Skin Friction Distribution on the Common Research Model," Paper 2011-1129, AIAA, January 2011.

${ }^{20}$ Rivers, M. and Hunter, G., "Support System Effects on the NASA Common Research Model," Paper 2012-0707, AIAA, January 2012 .

${ }^{21}$ Rivers, M., Hunter, G., and Campbell, R., "Further Investigation of the Support System Effects and Wing Twist on the NASA Common Research Model," Paper 2012-3209, AIAA, June 2012. 
${ }^{22}$ NASA, "Common Research Model," [online web site], http://commonresearchmodel.larc.nasa.gov/, 2012.

${ }^{23}$ Galle, M., "Ein Verfahren zur numerischen Simulation kompressibler, reibungsbehafteter Strömungen auf hybriden Netzen," Phd thesis, Uni Stuttgart, 1999.

${ }^{24}$ Kroll, N., Rossow, C.-C., Becker, K., and Thiele, F., "MEGAFLOW - A Numerical Flow Simulation System," Aerospace Science Technology, Vol. 4, 2000, pp. 223-237.

${ }^{25}$ Gerhold, T., "Overview of the Hybrid RANS Code TAU," MEGAFLOW, edited by N. Kroll and J. Fassbender, Vol. 89 of Notes on Numerical Fluid Mechanics and Multidisciplinary Design, Springer, 2005, pp. 81-92.

${ }^{26}$ Jameson, A., Schmidt, W., and Turkel, E., "Numerical Solution of the Euler Equations by Finite Volume Methods using Runge-Kutta Time Stepping Schemes," AIAA Paper 81-1259, Jan. 1981.

${ }^{27}$ Swanson, R. C. and Turkel, E., "On Central Differences and Upwind Schemes." Journal of Computational Physics, Vol. 101, pp., 1992.

${ }^{28}$ Heinrich, R., Wild, J., Streit, T., and Nagel, B., "Steady Fluid-Structure Coupling for Transport Aircraft," Onera-dlr aerospace symposium, Oct. 2006.

${ }^{29}$ Heinrich, R., Kroll, N., Neumann, J., and Nagel, B., "Fluid-Structure Coupling for Aerodynamic Analysis and Design A DLR Perspective," AIAA Paper 2008-0561, Jan. 2008

${ }^{30}$ Hounjet, M. and Meijer, J., "Evaluation of Elastomechanical and Aerodynamic Data Transfer Methods for Non-Planar Configurations in Computational Aeroelastic Analysis," Intern. forum on aeroelasticity and structural dynamics, manchester, uk, June 1995.

${ }^{31}$ Beckert, A. and Wendland, H., "Multivariante interpolation for fluid-structure interaction problems using radial basis functions," Aerospace Science and technology, Vol. 5, No. 2, pp. 125-134, 2001.

${ }^{32}$ Keye, S. and Rudnik, R., "Aero-Elastic Simulation of DLR's F6 Transport Aircraft Configuration and Comparison to Experimental Data," Paper 2009-0580, AIAA, Jan. 2009.

${ }^{33}$ Keye, S., "Fluid-Structure Coupled Analysis of a Transport Aircraft and Flight-Test Validation," AIAA Journal of Aircraft, 2011, Vol. 48, No. 2, 2011, pp. 381-390.

${ }^{34}$ Spalart, P. and Allmaras, S., "A One-Equation Turbulence Model for Aerodynamic-Flows," AIAA Paper 92-0439, 1992.

${ }^{35}$ Menter, F. R., "Two-Equation Eddy-Viscosity Turbulence Models for Engineering Applications," AIAA Journal, Vol. 32, No. 8, 1994, pp. 1598-1605

${ }^{36}$ Yamamoto, K., Tanaka, K., and Murayama, M., "Comparison Study of Drag Prediction for the 4th CFD Drag Prediction Workshop using Structured and Unstructured Mesh Methods," Paper 2010-4222, AIAA, June 2010.

${ }^{37}$ Yamamoto, K., Tanaka, K., and Murayama, M., "Effect of a Nonlinear Constitutive Relation for Turbulence Modeling on Predicting Flow Separation at Wing-Body Juncture of Transonic Commercial Aircraft," Paper 2012-2895, AIAA, June 2012.

${ }^{38}$ Sclafani, A., Vassberg, J., Winkler, C., Dorgan, A., Mani, M., Olsen, M., and Coder, J., "DPW-5 Analysis of the CRM in a Wing-Body Configuration Using Structured and Unstructured Meshes," AIAA Paper 2013-0048, 2013.

${ }^{39}$ Spalart, P., "Strategies for turbulence modelling and simulations," Tech. rep., Vol. 21, pp. 252-263, 2000.

40 "Experimental Data Base for Computer Program Assessment," AGARD-Report AGARD-R-138, 1979.

${ }^{41}$ Eisfeld, B. and Brodersen, O., "Advanced Turbulence Modelling and Stress Analysis for the DLR-F6 Configuration," Paper 2005-4727, AIAA, June 2005.

${ }^{42}$ Cécora, R.-D., Eisfeld, B., Probst, A., Crippa, S., and Radespiel, R., "Differential Reynolds Stress Modeling for Aeronautics," Paper 2012-0465, AIAA, January 2012.

${ }^{43}$ Durbin, P. A., "On the $k-3$ stagnation point anomaly," International Journal of Heat and Fluid Flow, Vol. 17, pp. 89-90, 1996.

${ }^{44}$ Wilcox, D. C., Turbulence Modeling for CFD, DCW Industries, 2nd ed., 1998.

${ }^{45}$ Vassberg, J., "A Unified Baseline Grid about the Common Research Model Wing-Body for the Fifth AIAA CFD Drag Prediction Workshop." Paper 2011-3508, AIAA, June 2011.

${ }^{46}$ CentaurSoft, "Centaur Hybrid Grid Generation System," [online web site], http://www.centaursoft.com, 2012.

${ }^{47}$ Martineau, D., Stokes, S., Munday, S., Jackson, A., Gribben, B., and Verhoeven, N., "Anisotropic Hybrid Mesh Generation for Industrial RANS Applications," AIAA Paper 2006-0534, Jan. 2006. 